\title{
Measuring the Cohesiveness of Preferences: An Axiomatic Analysis*
}

\author{
Jorge Alcalde-Unzu ${ }^{\dagger}$ and Marc Vorsatz ${ }^{\ddagger}$
}

June 2012

\begin{abstract}
In this paper, we axiomatically study how to measure the similarity of preferences in a group of individuals. For simplicity, we refer to this as the cohesiveness. First, we provide axioms that characterize a family of linear and additive measures whose intersection is a partial ordinal criterion similar to first order stochastic dominance. The introduction of some additional properties isolates a one-parameter subfamily. This parameter evaluates the effect on the cohesiveness if one individual changes his ranking on a single pair of objects, as a function of how many of the remaining individuals in the group rank the first object over the second and vice versa. Finally, we characterize the focal measures of this subfamily separately showing that they coincide with measures constructed using two, at first sight, totally different approaches suggested in the literature.
\end{abstract}

Keywords: Cohesiveness, characterization, distance, measure, cohesiveness, preferences.

JEL-Numbers: D39, D70.

${ }^{*}$ We are very grateful to the audiences in Barcelona, Bilbao, Montreal, Soria, and Vigo. We specially thank Katherine Baldiga, Miguel A. Ballester, Casilda Lasso de la Vega, Conchita D'Ambrosio, José Luis García-Lapresta, Jerry Green, Jordi Massó, Juan Moreno-Ternero, Jorge Nieto, David Pérez-Román, Ana Urrutia and two anonymous referees for for their valuable comments and suggestions.

${ }^{\dagger}$ Department of Economics, Public University of Navarre, Campus Arrosadia, 31006 Pamplona, Spain. Email: jorge.alcalde@unavarra.es. Financial support from the Spanish Ministry of Education and Science, through the projects ECO2009-11213 and ECO2009-12836, is gratefully acknowledged.

‡Corresponding author. Departamento de Análisis Económico II, Universidad Nacional de Educación a Distancia (UNED), Paseo Senda del Rey 11, 28040 Madrid, Spain and Fundación de Estudios de Economía Aplicada (FEDEA), Calle Jorge Juan 46, 28001 Madrid, Spain. Email: mvorsatz@cee.uned.es. Financial support from the Spanish Ministry of Education and Science, through the project ECO2009-07530, is gratefully acknowledged. 


\section{Introduction}

The cohesion in a group depends, among many other factors, on how similar its members are, where the similarity can be assessed using external characteristics such as age, ethnicity, income, or the access to opportunities and internal characteristics such as values, attitudes, or preferences (see, Eisenberg [12]). A significant body of the economic literature has worked on the axiomatic derivation of measures that evaluate the cohesion of a group in many of these dimensions - see, for example, Gini [16] and Atkinson [2] who, among others, propose measures of income inequality (also see Cowell [6] for an overview of this literature); Esteban and Ray [14] and Duclos et al. [9] who, among others, suggest ways to assess income, ethnic, or religious polarization; Hutchens [18] and Echenique and Fryer [10] who, among others, propose measures for quantifying the segregation of individual occupations and geographical districts; and, Kranich [22] and Roemer [24] who, among others, evaluate the distribution of opportunities among individuals. However, only a few studies have addressed the question of how to measure the similarity of preferences of the group members, one of the before-mentioned internal characteristics. For simplicity, we refer to this aspect from now on as the cohesiveness.

Bosch [5] was the first to define a cohesiveness measure as a function that assigns to each ranking profile - a list of ordinal preferences on an abstract set of objects, one ranking for each individual belonging to the group - a number from the unit interval. ${ }^{1}$ He also provides axiomatizations of some simple measures, for example the trivial measure that takes a value of one if all individuals have the same ranking, and a value of zero otherwise. After that, García-Lapresta and Pérez-Román [15] introduced several normative/operational properties, and studied whether some more elaborated measures satisfy these criteria. The objective of this work is to go one step further by providing closed axiomatic characterizations of some cohesiveness measures.

One intuitive way to construct a cohesiveness measure is to calculate first the similarity of preferences for each pair of individuals, and then aggregate these num-

\footnotetext{
${ }^{1} \mathrm{He}$ uses the term consensus instead of cohesiveness.
} 
bers by taking the average. Given that many distance measures between pairs of rankings have been studied in the literature, one can use them as indicators of dissimilarity and construct the cohesiveness measures around them. For example, the most well-known distance measure is probably Kemeny's one (see, Kemeny [20]) that calculates the proportion of pairwise comparisons the two rankings do not coincide upon. The cohesiveness between two individuals could then be measured by one minus the Kemeny distance - the proportion of pairwise comparisons the two rankings agree upon-, a measure of similarity that is usually referred to as Kendall's $\tau$ (see, Kendall [20]). The cohesiveness could then finally be obtained by calculating the average of the values of the $\tau$ measure over all possible pairs of individuals. This measure, called average tau $(\bar{\tau})$, was introduced by Hays [17].

Even though the described way of constructing a cohesiveness measure is reasonable and probably the most prominent one pursued in the literature, it has to be noted that it is also possible to construct a cohesiveness measure from a "dual" perspective: one determines first the degree to which the group agrees on how to order each pair of objects, and then aggregates these values by taking the average over all possible pairs of objects. This dual way of deriving a cohesiveness measure leads to a different measure. However, we will show in this paper by means of axiomatic characterizations that both approaches can be understood as focal cases of a general family that imports the concept of first order stochastic dominance to our setting.

We proceed as follows. In the next section, we introduce basic notation and definitions. Section 3 presents the first set of basic axioms and the characterization of a family of linear and additive measures $(\Omega)$. We also show that the partial ordering constructed by taking the intersection of all measures of $\Omega$ resembles the concept of first order stochastic dominance. Section 4 introduces some additional properties and provides the characterization of a subfamily $\Gamma$ of $\Omega$ that only depends on a single parameter. Section 5 characterizes the focal measures of $\Gamma$ and shows that these measures can be interpreted as the most natural measures of the two before-mentioned approaches. In fact, these characterizations identify a com- 
mon underlying structure of both approaches and the (surprisingly few) differences between them. We conclude in Section 6. Proofs and the independence of the properties used in the characterizations are relegated to the Appendix.

\section{Notation and definitions}

Consider a finite set of objects $X$ of cardinality $k \geq 2$ and a (countable) infinite set of individuals denoted by the set of natural numbers $\mathbb{N}$. Our objective is to evaluate the cohesiveness for all finite groups $N \subset \mathbb{N}$. We denote the size of the group $N$ by $n$ and we assume that $n \geq 2$. Elements of $X$ are usually denoted by $x, y$, and $z$, generic individuals are indexed by $i$ and $j$. We will also make frequent use of the letters $A, B$, and $C$ to denote groups. Let $\mathbb{Q}$ be the set of rational numbers. Given any rational number $r \in \mathbb{Q},\lfloor r\rfloor \in \mathbb{N}$ refers to the largest natural number smaller than or equal to $r$.

Let $P_{i}$ be the ranking of individual $i$ on $X$. We assume that $P_{i}$ is a complete, transitive, and antisymmetric binary relation. We denote the opposite ranking of $P_{i}$ by $P_{i}^{O P}$; that is, $x P_{i} y \Leftrightarrow y P_{i}^{O P} x$ for all $x, y \in X$. The set of all rankings on $X$ is denoted by $\mathcal{P}$. A profile $P=\left(P_{i}\right)_{i \in \mathbb{N}} \in \mathcal{P}^{\mathbb{N}}$ is a list of all individual rankings. Given a profile $P \in \mathcal{P}^{\mathbb{N}}$ and a group $N \subset \mathbb{N}$, a ranking profile $P_{N}=\left(P_{i}\right)_{i \in N} \in \mathcal{P}^{N}$ is an $n$-tuple of rankings, one for each individual belonging to $N$. We also say that two ranking profiles $P_{A} \in \mathcal{P}^{A}$ and $P_{B}^{\prime} \in \mathcal{P}^{B}$, corresponding to the groups $A$ and $B$ of equal size, are isomorphic whenever there exists a bijection $\pi: A \rightarrow B$ such that for all $i \in A, P_{i}=P_{\pi(i)}^{\prime}$.

Let $\bar{X}=\left\{\{x, y\} \in X^{2}: x \neq y\right\}$ be the set of all pairs of distinct objects; for example, if $X=\{x, y, z\}$, then $\bar{X}=\{\{x, y\},\{x, z\},\{y, z\}\}$. Given the ranking profile $P_{N} \in \mathcal{P}^{N}$ and a pair of objects $\{x, y\} \in \bar{X}, \#\left(x P_{N} y\right)=\#\left\{i \in N: x P_{i} y\right\}$ denotes the number of individuals who rank $x$ over $y$ at $P_{N}$. Then, $n_{x, y}\left(P_{N}\right)=$ $\left|\#\left(x P_{N} y\right)-\#\left(y P_{N} x\right)\right|$ is the absolute difference between the number of individuals who rank $x$ over $y$ and the ones who rank $y$ over $x$, always at $P_{N}$. For all ranking profiles $P_{N} \in \mathcal{P}^{N}$, let $d_{j}\left(P_{N}\right)=\frac{2}{k(k-1)}\left|\{x, y\} \in \bar{X}: n_{x, y}\left(P_{N}\right)=j\right|$ be the proportion of pairs of objects such that $n_{x, y}\left(P_{N}\right)$ is equal to $j$. It is easy to see 
that $d_{j}\left(P_{N}\right)$ may only take strictly positive values for $j=0,2, \ldots, n$ whenever the size of the group is even and for $j=1,3, \ldots, n$ whenever $n$ is odd. Consequently, the notation $d\left(P_{N}\right)=\left(d_{0}\left(P_{N}\right), d_{2}\left(P_{N}\right), \ldots, d_{n}\left(P_{N}\right)\right)$ is used for any even $n$ and $d\left(P_{N}\right)=\left(d_{1}\left(P_{N}\right), d_{3}\left(P_{N}\right), \ldots, d_{n}\left(P_{N}\right)\right)$ for any odd $n$.

Given the ranking $P_{i} \in \mathcal{P}$ and the pair of objects $\{x, y\} \in \bar{X}$, we will say that the ordered pair $(x, y)$ is a contiguous pair at $P_{i}$ if $x P_{i} y$ and there is no other object $z \in X$ such that $x P_{i} z P_{i} y$. For any two rankings $P_{i}, P_{i}^{\prime} \in \mathcal{P}$ and any pair of objects $\{x, y\} \in \bar{X}, P_{i}^{\prime}$ is said to be $(x, y)$-different from $P_{i}$ if $(y, x)$ is a contiguous pair at $P_{i},(x, y)$ is a contiguous pair at $P_{i}^{\prime}$, and $w P_{i} z \Leftrightarrow w P_{i}^{\prime} z$ for all pairs of objects $\{w, z\} \in(\bar{X} \backslash\{\{x, y\}\})$. Finally, given the ranking profiles $P_{N}, P_{N}^{\prime} \in \mathcal{P}^{N}$, an individual $i \in N$, and a pair of objects $\{x, y\} \in \bar{X}, P_{N}^{\prime}$ is said to be $(x, y)-$ different from $P_{N}$ for individual $i$ if $P_{i}^{\prime}$ is $(x, y)$-different from $P_{i}$ and $P_{j}^{\prime}=P_{j}$ for all $j \notin(N \backslash\{i\})$. Intuitively, $P_{N}^{\prime}$ is $(x, y)$-different from $P_{N}$ for individual $i$ if $P_{N}^{\prime}$ can be derived from $P_{N}$ by only reversing the binary relation $y P_{i} x$. In this case, we will also say that $P_{N}^{\prime}$ can be obtained from $P_{N}$ by means of a $y P_{i} x-$ change.

Given a set of individuals $N \subset \mathbb{N}$, a cohesiveness measure for group $N$ is a function $M^{N}: \mathcal{P}^{N} \rightarrow[0,1]$ that assigns to every ranking profile $P_{N} \in \mathcal{P}^{N}$ a real number $M\left(P_{N}\right)$ from the unit interval. For notational purposes, we will write $M\left(P_{N}\right)$ instead of $M^{N}\left(P_{N}\right)$. A cohesiveness measure $M$ is a family of measures $\left\{M^{N}: \mathcal{P}^{N} \rightarrow[0,1]\right\}_{N \subset \mathbb{N}}$.

\section{A linear and additive family of measures}

We present four basic properties and show that they fully characterize a general family of linear and additive measures. The first property, Anonymity, states that all individuals are equally important in determining the cohesiveness. Formally, this idea is modeled by requiring the cohesiveness measure to take equal values for any two isomorphic ranking profiles. This property was included directly in the definition of a cohesiveness measure by Bosch [5] and García-Lapresta and PérezRomán [15], but we prefer to consider it as an independent property. 
ANONYMITY (AN): The cohesiveness measure $M$ is anonymous if for all groups $A, B \subset \mathbb{N}$ and all isomorphic ranking profiles $P_{A} \in \mathcal{P}^{A}$ and $P_{B}^{\prime} \in \mathcal{P}^{B}$,

$$
M\left(P_{B}^{\prime}\right)=M\left(P_{A}\right)
$$

The second property, Independence, states that the effect on the cohesiveness of a $y P_{i} x$-change is independent of all other pairwise comparisons. To express this more formally, consider any two ranking profiles $P_{N}$ and $\bar{P}_{N}$ with the property that no individual changes her ranking on the pair $\{x, y\}$ across the two situations. Suppose also that $(y, x)$ is a contiguous pair of objects for $i$ at both ranking profiles. Consider now the two ranking profiles $P_{N}^{\prime}$ and $\bar{P}_{N}^{\prime}$ that are obtained from $P_{N}$ and $\bar{P}_{N}$, respectively, by changing the ordering between $x$ and $y$ for individual $i$. Then, the cohesiveness should change in both situations by the same amount.

INDEPENDENCE (IND): The cohesiveness measure $M$ is independent if for all groups $N \subset \mathbb{N}$, all individuals $i \in N$, all pairs of distinct objects $\{x, y\} \in \bar{X}$, and all ranking profiles $P_{N}, P_{N}^{\prime}, \bar{P}_{N}, \bar{P}_{N}^{\prime} \in \mathcal{P}^{N}$ such that $x P_{j} y \Leftrightarrow x \bar{P}_{j} y$ for all $j \in N$, $P_{N}^{\prime}$ is $(x, y)$-different from $P_{N}$ for individual $i$, and $\bar{P}_{N}^{\prime}$ is $(x, y)$-different from $\bar{P}_{N}$ for individual $i$,

$$
M\left(\bar{P}_{N}^{\prime}\right)-M\left(\bar{P}_{N}\right)=M\left(P_{N}^{\prime}\right)-M\left(P_{N}\right)
$$

The third property, Neutrality, states that the cohesiveness measure is not biased with respect to certain objects. Formally, if it is possible to derive one ranking profile from a distinct one by only relabeling objects, then the cohesiveness should be the same in both situations. This property was also included directly in the definition of a cohesiveness measure by Bosch [5] and García-Lapresta and Pérez-Román [15].

NEUTRALITY (NEU): The cohesiveness measure $M$ is neutral if for all groups $N \subset \mathbb{N}$ and all ranking profiles $P_{N}, P_{N}^{\prime} \in \mathcal{P}^{N}$ such that there exists a permutation $\mu: X \rightarrow X$ with the property that for all individuals $i \in N$ and all pairs of distinct objects $\{x, y\} \in \bar{X}, x P_{i} y \Leftrightarrow \mu(x) P_{i}^{\prime} \mu(y)$,

$$
M\left(P_{N}^{\prime}\right)=M\left(P_{N}\right)
$$


The last property, Monotonicity, regards the situation when all individuals agree on how to rank all pairs of objects apart from $\{x, y\}$ and a single individual $i$ performs a $y P_{i} x$-change. Then, if the number of individuals in the rest of the group who rank $x$ over $y$ is greater than the number of individuals who rank $y$ over $x$, the cohesiveness should increase. Monotonicity therefore highlights that the effect on the cohesiveness of interchanging a contiguous pair of objects when there is unanimity in the rest of comparisons should depend on the rankings the other members of the group have on that pair of objects. In particular, the sign of the change in the cohesiveness depends on which of the two objects is preferred by the majority of individuals.

MONOTONICITY $(\mathrm{MON})$ : The cohesiveness measure $M$ is monotonic if for all groups $N \subset \mathbb{N}$, all individuals $i \in N$, all pairs of distinct objects $\{x, y\} \in \bar{X}$, and all ranking profiles $P_{N}, P_{N}^{\prime} \in \mathcal{P}^{N}$ such that $w P_{j} z \Leftrightarrow w P_{l} z$ for all $\{w, z\} \in(\bar{X} \backslash\{\{x, y\}\})$ and all $j, l \in N$, and $P_{N}^{\prime}$ is $(x, y)$-different from $P_{N}$ for individual $i$,

$$
\#\left(x P_{N \backslash\{i\}} y\right)>\#\left(y P_{N \backslash\{i\}} x\right) \Rightarrow M\left(P_{N}^{\prime}\right)>M\left(P_{N}\right) .
$$

Theorem 1 shows that these properties fully characterize a family of linear and additive cohesiveness measures with the vector of the absolute differences $d\left(P_{N}\right)$ as main component. Note that because of Anonymity, the vector of weights $a^{n}$ depends on the size $n$ of the group $N$ and not on the group itself.

Theorem 1 The cohesiveness measure $M$ satisfies AN, IND, NEU, and MON if and only if for all groups $N \subset \mathbb{N}$, there exists a vector $a^{n}=\left(a_{1}^{n}, a_{2}^{n}, \ldots, a_{\left\lfloor\frac{n}{2}\right\rfloor+1}^{n}\right)$, with $a_{i}^{n} \in[0,1]$ for all $i \in\left\{1,2, \ldots,\left\lfloor\frac{n}{2}\right\rfloor+1\right\}$ and $a_{j}^{n}<a_{j+1}^{n}$ for all $j \in\left\{1,2, \ldots,\left\lfloor\frac{n}{2}\right\rfloor\right\}$, such that for all ranking profiles $P_{N} \in \mathcal{P}^{N}$,

$$
M\left(P_{N}\right)=a^{n} \cdot d\left(P_{N}\right)
$$

We denote the family of cohesiveness measures characterized in Theorem 1 by $\Omega$. We conclude this section with a graphical representation showing that the intersection of all measures belonging to $\Omega$ establishes a partial order on ranking profiles of the same size that is of the same nature as first order stochastic dominance. The following example will illustrate this point. 
Suppose that $n=8$ and $k=5$ so that, in total, there are ten different pairs of objects. In Figure 1, the horizontal axis refers to the indexes of the vector $d$, which are $0,0.25,0.5,0.75$ and 1 . The vertical axis, on the other hand, collects cumulative percentages of $d$ represented by the function $D\left(P_{N}, j\right)$. This function assigns to any ranking profile $P_{N} \in \mathcal{P}^{N}$ and any number $j \in\{0,0.25,0.5,0.75,1\}$, the value $D\left(P_{N}, j\right)=\sum_{i<j} d_{i}\left(P_{N}\right)$. For example, if $D\left(P_{N}, 0.25\right)=0.6$, as it is the case of profile $3, n_{x, y}\left(P_{N}\right)$ is smaller than or equal to $0.25 \cdot 8=2$, for sixty percent of all possible pairs of objects.

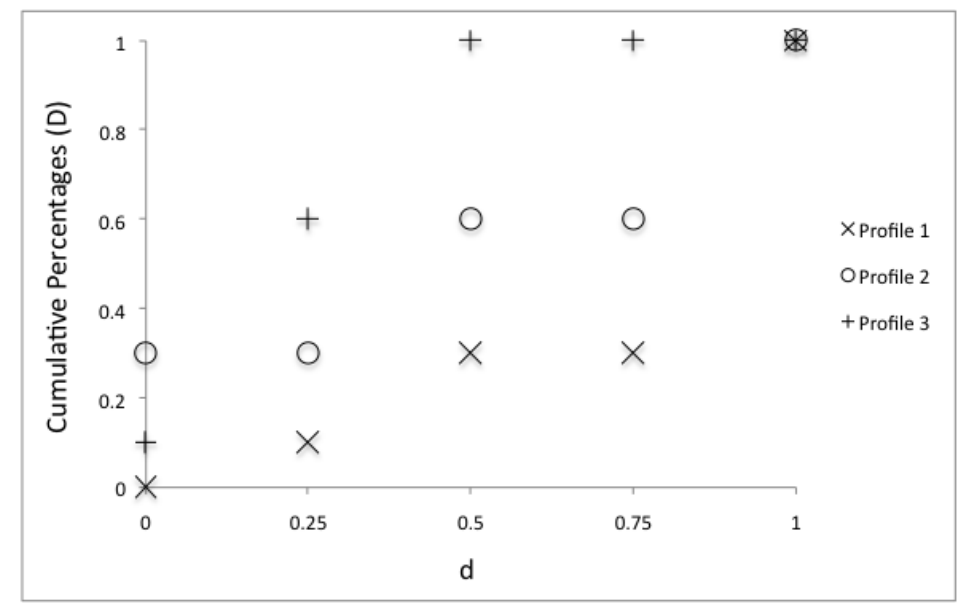

Figure 1: Dominance criterion.

Now, if the values of $D$ of one ranking profile lie never above and in some case strictly below the ones of a second ranking profile, all measures belonging to $\Omega$ assign a higher cohesiveness to the former profile. Thus, given the three ranking profiles in Figure 1, the cohesiveness in profile 1 is higher than in profiles 2 and 3 for all measures in $\Omega$. Note also the partial ordering induced by the intersection of all the measures of the family $\Omega$ does not put any restriction on which ranking profile has the higher cohesiveness whenever the values of $D$ cross in some instance (i.e., for profiles 2 and 3 ).

There are many ways to complete the partial order induced by $\Omega$. One approach is to calculate, for any two ranking profiles, the distances between the values of $D$. The distances are then aggregated in order to decide which of the two ranking 
profiles has the higher cohesiveness. Interestingly, this measure coincides with the one constructed using one of the approaches explained in the introduction. This correspondence and the exact formulation of the measure will be provided in Section 5 .

\section{A one-parameter subfamily of linear and addi- tive measures}

Theorem 1 shows that a set of intuitive axioms restricts the way in which the cohesiveness should be measured; it imposes a linear and additive formula over the majority results of all pairwise comparisons over objects. In addition, it also induces some ordinal comparisons between ranking profiles of the same size. However, the family $\Omega$ is rather large and includes measures that suffer from several shortcomings. In particular:

1. So far, the structure introduced is not sufficient to establish an undoubtful comparison between ranking profiles of different sizes, even in ordinal terms. For example, $\Omega$ contains measures that assign less cohesiveness to an unanimous ranking profile of a group with size $n$ than to a ranking profile from individuals with very different rankings in a group of size $n+1$. Some structure on the values of a cohesiveness measure for ranking profiles of different sizes is needed.

2. A particular characteristic of the family $\Omega$ is that the exact cardinal values are not determined. For example, some cohesiveness measures of $\Omega$ only take values between 0 and $\varepsilon$ (for any $\varepsilon>0$ ), whereas others only take values between $1-\varepsilon$ and 1 . $\Omega$ even includes measures that take values between 0 and $\varepsilon$ for some group sizes and values between $1-\varepsilon$ and 1 for others. This shows why some homogeneous structure is needed so that the value assigned to a ranking profile has some meaning per se.

3. According to Theorem 1 , a $y P_{i} x$-change increases the cohesiveness if at least 
as many individuals rank $x$ over $y$ as the other way round. It also allows the quantity of this increase to depend on the size of the majority. However, $\Omega$ includes measures that evaluate these effects arbitrarily, for example depending on whether or not the majority in a given ranking profile is a prime number. Hence, some consistency in the quantity changes is asked for.

These shortcomings motivate us to propose additional axioms in order to further restrict $\Omega$. The first axiom, Replication Invariance, puts some structure on ranking profiles belonging to groups of different sizes. Suppose that we are able to assess the cohesiveness for groups of size $n$. Then, one can consider a sequence of ranking profiles of size $n$. If it turns out that all ranking profiles belonging to the sequence are isomorphic copies of each other, then the ranking profile that consists of the union of all isomorphic copies has the same preference structure as each of its components. Then, a basic coherence property requires that the cohesiveness in this augmented ranking profile is the same as in any of its components (see Blackorby et al. [4] for applications of this type of property in other social choice contexts).

REPLICATION INVARIANCE (REP): The cohesiveness measure $M$ satisfies replication invariance if for all ranking profiles $P_{A} \in \mathcal{P}^{A}$ and $P_{B}^{\prime} \in \mathcal{P}^{B}$, where $|B|=m \cdot|A|($ with $m \in \mathbb{N})$, such that $P_{B}^{\prime}$ consists of the union of $m$ isomorphic and disjoint copies of $P_{A}$,

$$
M\left(P_{B}^{\prime}\right)=M\left(P_{A}\right)
$$

Replication Invariance reduces to Anonymity if $m=1$. Intuitively, it implies that the cohesiveness measure does not focus on the absolute number of individuals having each possible ranking, but on the proportions.

To fill the second gap and construct homogeneous cohesiveness measures, we require that the extreme values a cohesiveness measure can reach by definition (0 and 1) are attained for some ranking profiles (corresponding to possibly different groups of possibly different sizes). This property serves the objective that the value a cohesiveness measure assigns to a ranking profile provides some information per se. 
FULL RANGE (FR): The cohesiveness measure $M$ satisfies full range if there are two groups $A$ and $B$ and two ranking profiles $P_{A} \in \mathcal{P}^{A}$ and $P_{B}^{\prime} \in \mathcal{P}^{B}$ such that

$$
M\left(P_{A}\right)=0 \text { and } M\left(P_{B}^{\prime}\right)=1
$$

To introduce our next axiom, Concordance, consider a ranking profile $P_{N}$ such that three individuals $i, j, l \in N$ have a contiguous pair $(y, x)$ in their rankings, but there is a majority of individuals who rank $x$ over $y$. Also, all individuals agree on how to order all pairs of objects different from $\{x, y\}$. Now, suppose that individual $i$ changes her/his ranking over the pair $\{x, y\}$. This $y P_{i} x$-change could cause the cohesiveness to vary (in particular, because of MON it increases the cohesiveness for any measure of $\Omega$ ). Suppose now that after this $y P_{i} x$-change, $j$ also changes her ranking on $\{x, y\}$. As a result, the cohesiveness could change again. Finally, if $l$ also switches her ranking, the cohesiveness could change again. However, it seems natural that these changes (if they exist) are related to each other in some way. For example, if the second change causes the cohesiveness to vary in the same direction and quantity as the first one, then there are not apparent reasons why the third quantity change should be different from the first two. If, however, the second change increases the cohesiveness by a larger (smaller) quantity than the first one, then it seems natural that the third change increases the cohesiveness by this amount more (less) than the second one.

CONCORDANCE (CON): The cohesiveness measure $M$ is concordant if for all groups $N \subset \mathbb{N}$, all individuals $i, j, l \in N$, all pairs of distinct objects $\{x, y\} \in \bar{X}$, and all ranking profiles $P_{N}, P_{N}^{\prime}, P_{N}^{\prime \prime}, P_{N}^{\prime \prime \prime} \in \mathcal{P}^{N}$ such that $w P_{s} z \Leftrightarrow w P_{m} z$ for all $\{w, z\} \in(\bar{X} \backslash\{\{x, y\}\})$ and all $s, m \in N, P_{N}^{\prime}$ is $(x, y)$-different from $P_{N}$ for $i$, $P_{N}^{\prime \prime}$ is $(x, y)$-different from $P_{N}^{\prime}$ for $j, P_{N}^{\prime \prime \prime}$ is $(x, y)$-different from $P_{N}^{\prime \prime}$ for $l$, and $\#\left(x P_{N} y\right) \geq \#\left(y P_{N} x\right)$,

$\left(M\left(P_{N}^{\prime}\right)-M\left(P_{N}\right)\right)-\left(M\left(P_{N}^{\prime \prime}\right)-M\left(P_{N}^{\prime}\right)\right)=\left(M\left(P_{N}^{\prime \prime}\right)-M\left(P_{N}^{\prime}\right)\right)-\left(M\left(P_{N}^{\prime \prime \prime}\right)-M\left(P_{N}^{\prime \prime}\right)\right)$

Adding FR and CON to the former properties and replacing AN by REP characterizes a one-parameter subfamily of $\Omega$. 
Theorem 2 The cohesiveness measure $M$ satisfies IND, NEU, MON, REP, FR, and $C O N$ if and only if $M \in \Omega$ and there exists $\gamma \in\left[\frac{1}{4}, \frac{3}{4}\right]$ such that for all $n \geq 2$ and all $i \in\left\{1, \ldots,\left\lfloor\frac{n}{2}\right\rfloor+1\right\}$,

$$
a_{i}^{n}= \begin{cases}\frac{2(i-1)}{n^{2}}\left[4\left(\gamma-\frac{1}{4}\right) n+\left(1-4\left(\gamma-\frac{1}{4}\right)\right) 2(i-1)\right] & \text { if } n \text { is even } \\ \frac{2 i-1}{n^{2}}\left[4\left(\gamma-\frac{1}{4}\right) n+\left(1-4\left(\gamma-\frac{1}{4}\right)\right)(2 i-1)\right] & \text { if } n \text { is odd. }\end{cases}
$$

We denote the family of cohesiveness measures characterized in Theorem 2 by $\Gamma$. With respect to the parameter $\gamma$, it follows from the proof that it is exactly equal to $a_{2}^{4}$. Hence, its value is related to the question of how much cohesiveness there is in a group of four individuals if, for all pairs of objects, three individuals rank the first object over the second and one individual the second over the first. Also, we knew by Theorem 1 that any change of a contiguous pair of objects produces a variation in any cohesiveness measure of $\Omega$ whose sign depends on which object is ranked higher by the majority of the remaining individuals. However, the magnitude of this variation was allowed to depend on the size of the majority and it is this dependence that is now uniquely captured through the parameter $\gamma$. If $\gamma=0.5$, all changes are considered equally important; that is, the cohesiveness measure associated with this value ignores the size of the majority when judging changes of contiguous pairs. If $\gamma>0.5$, the effect on the cohesiveness of a change in a contiguous pair is the higher the more divided the group is on that particular pair of objects (and this effect is the more pronounced the higher $\gamma$ is). Finally, if $\gamma<0.5$, a change of a contiguous pair gets a higher weight the less divided the group is on these objects (and this effect is the more pronounced the smaller $\gamma$ is).

\section{The measures of $\Gamma$}

We will now introduce additional properties and show that when they are added to the ones of Theorem 2, the measures corresponding to $\gamma=0.25$ and $\gamma=0.5$ are characterized. These measures have not only a special interest because they are focal in the family $\Gamma$, but also because they have an intuitive interpretation in terms of the two approaches to construct cohesiveness measures mentioned in the 
introduction. We will also see that the rest of measures of $\Gamma$ can be related to these two focal cases.

\subsection{Corrected normalized average tau}

To introduce the first additional property, we will focus on some correlation measures that have received a particular attention in the literature. For the special case of two individuals, Kendall [19] has proposed a well-known correlation measure that is based on the distance measure of Kemeny [20]. To define it formally, take any group of two individuals $N=\{i, j\}$ and any ranking profile $P_{N} \in \mathcal{P}^{N}$ of this group as given. Now, for any pair of objects $\{x, y\} \in \bar{X}, \tau_{i, j}^{x, y}\left(P_{N}\right)=1$ if individual $i$ and $j$ agree on the binary ordering between $x$ and $y$, otherwise $\tau_{i, j}^{x, y}\left(P_{N}\right)=0$. The cohesiveness in this group, $\tau_{i, j}\left(P_{N}\right)$, is then defined as the proportion of pairwise comparisons that both individuals agree upon; that is, $\tau_{i, j}\left(P_{N}\right)=\frac{2}{k(k-1)} \sum_{\{x, y\} \in \bar{X}} \tau_{i, j}^{x, y}\left(P_{N}\right)$. For the general case of any group $N \subset \mathbb{N}$ of size $n$, Hays [17] suggests to naturally extend Kendall's $\tau$ by assigning to any ranking profile $P_{N} \in \mathcal{P}^{N}$ the average of all possible $\tau_{i, j}\left(P_{N}\right)$ values; that is, $\bar{\tau}\left(P_{N}\right)=\frac{2}{n(n-1)} \sum_{i, j \in N: j \neq i} \tau_{i, j}\left(P_{N}\right)$. This measure is referred to as average tau. Since the extreme values of $\bar{\tau}\left(P_{N}\right)$ for a given group $N$ may be different from 0 and 1, Hays [17] also proposes to normalize the measure to the unit interval. The normalized average tau is given by $\tilde{\tau}\left(P_{N}\right)=\frac{\bar{\tau}\left(P_{N}\right)-\min \left\{\bar{\tau}^{N}\right\}}{\max \left\{\bar{\tau}^{N}\right\}-\min \left\{\bar{\tau}^{N}\right\}}$, taking into account that $\max \left\{\bar{\tau}^{N}\right\}=1, \min \left\{\bar{\tau}^{N}\right\}=\frac{n-2}{2(n-1)}$ if $n$ is even, and $\min \left\{\bar{\tau}^{N}\right\}=\frac{n-1}{2 n}$ if $n$ is odd. ${ }^{2}$

We have argued before that all measures belonging to $\Gamma$ only differ in how the cohesiveness varies as a response to a $y P_{i} x$-change. This magnitude is a function of $n_{x, y}$. Given the importance of $\bar{\tau}$ and $\tilde{\tau}$ in the literature, we are thus interested in studying how they behave in this aspect. The answer to this question will provide us with an idea of a new property that allows us to discriminate between the measures belonging to $\Gamma$.

By definition, $\bar{\tau}$ and $\tilde{\tau}$ evaluate a change of a contiguous pair depending on the variations in the distances between the ranking of this individual and the rankings of

\footnotetext{
${ }^{2}$ Lemma 1 of Alcalde-Unzu and Vorsatz [1] calculates these maximum and minimum values.
} 
each of the other individuals. To be more exact, a $y P_{i} x$-change produces two effects on $\bar{\tau}$ and $\tilde{\tau}$ : it reduces the distance (and, thus, increases the cohesiveness) between the ranking of this individual $i$ and the individuals who rank the object that is made better off higher; simultaneously, it increases the distance (and, thus, reduces the cohesiveness) between the ranking of $i$ and the individuals who rank the object that is made worse off higher. As a result, the increase in the cohesiveness changes proportionally with the number of individuals who rank the object that moves up in the ranking higher. Consequently, we can formally express this characteristic of $\bar{\tau}$ and $\tilde{\tau}$ by the following property, which is stronger than IND and CON.

PROPORTIONALITY (PROP): The cohesiveness measure $M$ is proportional if for all groups $N \subset \mathbb{N}$, all individuals $i \in N$, all pairs of distinct objects $\{x, y\} \in \bar{X}$, and all ranking profiles $P_{N}, P_{N}^{\prime}, \bar{P}_{N}, \bar{P}_{N}^{\prime} \in \mathcal{P}^{N}$ such that $P_{N}^{\prime}$ is $(x, y)$-different from $P_{N}$ for individual $i, \bar{P}_{N}^{\prime}$ is $(x, y)$-different from $\bar{P}_{N}$ for individual $i$, \# $\left(x P_{N} y\right) \geq$ $\#\left(y P_{N} x\right)$, and $\#\left(x \bar{P}_{N} y\right) \geq \#\left(y \bar{P}_{N} x\right)$,

$$
\frac{M\left(P_{N}^{\prime}\right)-M\left(P_{N}\right)}{M\left(\bar{P}_{N}^{\prime}\right)-M\left(\bar{P}_{N}\right)}=\frac{n_{x, y}\left(P_{N \backslash\{i\}}\right)}{n_{x, y}\left(\bar{P}_{N \backslash\{i\}}\right)} .
$$

Given that $\bar{\tau}$ and $\tilde{\tau}$ satisfy PROP and that they are constructed using pairwise comparisons, one would think that adding PROP to the former properties leads to a characterization of one of these measures. However, this is not true. The reason is that these measures, although they satisfy the other axioms, do not satisfy REP. To see why, consider the ranking profile $P_{\{1,2,3\}}$ such that $P_{1}=P_{2}=P_{3}^{O P}$. Consider now an extension of this ranking profile obtained by joining it with an isomorphic and disjoint ranking profile; that is, consider the ranking profile $P_{\{1,2,3,4,5,6\}}$ such that $P_{1}=P_{2}=P_{4}=P_{5}=P_{3}^{O P}=P_{6}^{O P}$. REP implies that the cohesiveness is in both situations the same. However, $\bar{\tau}\left(P_{\{1,2,3\}}\right)=\frac{1}{3}$ and $\bar{\tau}\left(P_{\{1,2,3,4,5,6\}}\right)=\frac{7}{15}$, whereas $\tilde{\tau}\left(P_{\{1,2,3\}}\right)=0$ and $\tilde{\tau}\left(P_{\{1,2,3,4,5,6\}}\right)=\frac{1}{9}$.

Consequently, given the intuitive plausibility of PROP, the following question arises: Which of the measures belonging to $\Gamma$ satisfies PROP (if there is any)? We are going to see that the unique measure is the one corresponding to $\gamma=0.25$. Curiously, it coincides with $\tilde{\tau}$ for even groups, but it corrects it for odd groups 
in such a way that the deviation tends to zero as the size of the group goes to infinity. In particular, for any ranking profile $P_{N}$ belonging to a group $N$ with an odd number of individuals, the measure can be calculated as $\frac{1}{n^{2}}+\tilde{\tau}\left(P_{N}\right) \frac{n^{2}-1}{n^{2}}$.

Definition 1 The cohesiveness measure $M$ is called corrected normalized average tau $(\hat{\tau})$ if $M \in \Gamma$ and $\gamma=0.25$ or, equivalently, if for all $n \geq 2$ and all $j \in$ $\left\{1, \ldots,\left\lfloor\frac{n}{2}\right\rfloor+1\right\}$,

$$
a_{i}^{n}= \begin{cases}\left(\frac{2(i-1)}{n}\right)^{2} & \text { if } n \text { is even } \\ \left(\frac{2 i-1}{n}\right)^{2} & \text { if } n \text { is odd }\end{cases}
$$

Replacing IND and CON by PROP isolates $\hat{\tau}$ from all measures in $\Gamma$.

Theorem 3 The cohesiveness measure $M$ satisfies REP, NEU, PROP, MON, and FR if and only if it is $\hat{\tau}$.

\subsection{Average sigma}

While the measures in the previous subsection calculate first the distances between individual rankings, an alternative approach determines first the distances in pairwise comparisons of objects. If there are only two objects -i.e., $X=\{x, y\}-$ the approach consists in calculating the absolute difference in the support of the two objects and weighting it by the maximal difference possible; that is, for all $N \subset \mathbb{N}$ and all ranking profiles $P_{N} \in \mathcal{P}^{N}, \sigma_{x, y}\left(P_{N}\right)=\frac{n_{x, y}\left(P_{N}\right)}{n}$. This measure can be naturally extended to the case of an arbitrary number of objects by following the reasoning of Hays [17] and calculating the average of all $\sigma_{x, y}\left(P_{N}\right)$ for all pairs of objects; that is, $\bar{\sigma}\left(P_{N}\right)=\frac{2}{k \cdot(k-1)} \sum_{\{x, y\} \in \bar{X}} \sigma_{x, y}\left(P_{N}\right)$. We will call this measure average sigma. As before, given the intuitive plausibility of $\bar{\sigma}$, we would like to know how it behaves in the aspect that differentiates the measures of $\Gamma$. It is easy to see that, if the majority of the individuals rank $x$ over $y$, any $y P_{i} x$-change induces $\bar{\sigma}$ to vary in the same way, independently of the size of the majority. Formally, this can be expressed as a property that states that the effect of interchanging a contiguous pair of objects only depends on which object was ranked higher by the majority before the change in the ranking took place. 
STRONG INDEPENDENCE (SIND): The cohesiveness measure $M$ is strongly independent if for all groups $N \subset \mathbb{N}$, all individuals $i \in N$, all pairs of distinct objects $\{x, y\} \in \bar{X}$, and all ranking profiles $P_{N}, P_{N}^{\prime}, \bar{P}_{N}, \bar{P}_{N}^{\prime} \in \mathcal{P}^{N}$ such that $P_{N}^{\prime}$ is $(x, y)-$ different from $P_{N}$ for individual $i, \bar{P}_{N}^{\prime}$ is $(x, y)$-different from $\bar{P}_{N}$ for individual $i$, $\#\left(x P_{N} y\right) \geq \#\left(y P_{N} x\right)$, and $\#\left(x \bar{P}_{N} y\right) \geq \#\left(y \bar{P}_{N} x\right)$,

$$
M\left(\bar{P}_{N}^{\prime}\right)-M\left(\bar{P}_{N}\right)=M\left(P_{N}^{\prime}\right)-M\left(P_{N}\right) .
$$

It can be easily checked that SIND implies IND and CON. The following result shows that the only measure of $\Gamma$ that satisfies SIND coincides with $\gamma=0.5$, which is exactly $\bar{\sigma}$.

Theorem 4 The following statements are equivalent:

1. $M$ satisfies REP, SIND, NEU, MON, and FR.

2. $M \in \Gamma$ and $\gamma=0.5$.

3. $M \in \Gamma$ and for all $n \geq 2$ and all $j \in\left\{1, \ldots,\left\lfloor\frac{n}{2}\right\rfloor+1\right\}$,

$$
a_{i}^{n}= \begin{cases}\frac{2(i-1)}{n} & \text { if } n \text { is even } \\ \frac{2 i-1}{n} & \text { if } n \text { is odd. }\end{cases}
$$

4. $M$ is $\bar{\sigma}$.

Finally, we would like to note that average sigma has a natural interpretation in terms of Figure 1. It implies that a complete ordering on ranking profiles of groups with the same size is obtained by summing up the distances in the values of the function $D$.

\subsection{Other measures of $\Gamma$}

The remaining measures of $\Gamma$ do not have a natural interpretation, nevertheless we can relate them to the focal cases studied before. First, the polar measure that corresponds to $\gamma=0.75$ can be obtained by proposing an inverse property to PROP such that the right-hand side of the property changes to $\frac{n_{x, y}\left(\bar{P}_{N \backslash\{i\}}\right)}{n_{x, y}\left(P_{N \backslash\{i\}}\right)}$. Second, the measures corresponding to parameter values between 0.25 and 0.5 are weighted 
averages of these two extremes, with weights depending on the distance between the parameter value of the measure and these extremes. ${ }^{3}$ Exactly, any measure $M_{\gamma}$ of the family $\Gamma$, where $\gamma \in\left[\frac{1}{4}, \frac{1}{2}\right]$, can be written as

$$
M_{\gamma}=4\left(\gamma-\frac{1}{4}\right) \cdot \bar{\sigma}+\left(1-4\left(\gamma-\frac{1}{4}\right)\right) \cdot \hat{\tau}
$$

That is, all these measures of the family are the different compromises between the ideas of PROP and SIND of these two extremes.

Finally, this equivalence has a mirror counterpart with the measures whose parameter values are between 0.5 and 0.75 .

\section{Concluding discussion}

Group cohesiveness can be defined as the degree to which the members of the group stick together, both emotional and task-related. If we think, for example, of an organizational unit at the workplace, group cohesiveness plays a central role not only because it leads to a better performance (i.e., there is a higher likelihood that a goal is met and/or the quality of the output is higher), but also because it decreases the probability of internal conflicts.

The objective of this study has been to measure the cohesiveness of individuals in a group on one particular dimension: preferences. We have first introduced a set of basic properties (AN, IND, NEU and MON) and have shown that they fully characterize a family of linear and additive measures $\Omega$ that takes the vector of distances $d$ as main component. In the next step of our study, we have used the properties of REP, FR and CON to restrict $\Omega$ further. In particular, the three additional properties imply that the set of weighting vectors $a^{n}$ depends on a single parameter $\gamma$, which determines the extend to which the size of the majority in a pairwise comparison affects the changes in the cohesiveness. Finally, we have established that the focal cases of $\Gamma$ have natural explanations in terms of two natural approaches to construct cohesiveness measures.

\footnotetext{
${ }^{3}$ We thank an anonymous referee for drawing our attention to this discussion.
} 
All measures characterized in the paper have in common that the unanimous ranking profiles (i.e., all individuals have exactly the same ranking) are the only profiles for which the cohesiveness is maximal. This is totally uncontroversial. In fact, Bosch [5] and García-Lapresta and Pérez-Román [15] impose this condition as part of the definition of a cohesiveness measure. The selection of the ranking profiles with the minimal cohesiveness, on the other hand, is not as clear cut. ${ }^{4}$ Consider, for example, two ranking profiles $P_{N}$ and $\bar{P}_{N}$ such that individual preferences are equally distributed over all possible preferences in $P_{N}$, but under $\bar{P}_{N}$, half of the population has a given ranking $\bar{P}_{i}$ and the other half has exactly the opposite ranking $\bar{P}_{i}^{O P}$. Given that $n_{x, y}\left(P_{N}\right)=0$ and $n_{x, y}\left(\bar{P}_{N}\right)=0$ for all $x, y \in X, P_{N}$ and $\bar{P}_{N}$ have both the lowest possible cohesiveness for any measure we characterize. Yet, there are arguments to defend other possibilities.

On one hand, one can argue that the cohesiveness should be lower under $P_{N}$ than under $\bar{P}_{N}$. After all, in $\bar{P}_{N}$ there are two subgroups in the society that each have full cohesiveness, although these subgroups are opposed to each other. On the other hand, one can also argue that $\bar{P}_{N}$ reflects a much more polarized society than $P_{N}$ and this higher polarization can lead to more conflicts. Therefore, it seems important to study in the future measures that can possibly differentiate between ranking profiles such as $P_{N}$ and $\bar{P}_{N}$. For example, it might be interesting to consider measures of polarization that explicitly consider intra-group cohesiveness and intergroup distances.

Finally, we would like to mention that our work contributes to an extensive literature in social choice in which distance measures play an important role. For example, there are papers that study or construct voting rules using distance measures (see, among others, Eckert et al. [11], Elkind et al. [13] and Meskanen and Nurmi [23]). With respect to the construction of distance measures, the results in our paper directly relate to the seminal work of Kemeny [20]. However, alternative distance measures have been proposed, for example, by Cook and Seiford $[7,8]$ and, more recently, by Klamler [21] and Baldiga and Green [3]. The former authors sug-

\footnotetext{
${ }^{4}$ We thank an anonymous referee for drawing our attention to this discussion.
} 
gest to sum the differences in the positions of each object in the two rankings and divide the resulting number by the maximal possible value of this sum. Klamler [21] and Baldiga and Green [3], on the other hand, considers all possible subsets (with a size of at least two) generated by the set of objects and calculates the proportion of different maximal objects between the two rankings. An interesting area of further research is the study of the properties of the corresponding cohesiveness measures that can be constructed using these distances in the same straightforward way as $\bar{\tau}$ and $\tilde{\tau}$ are constructed from the Kemeny distance through Kendall's $\tau$.

\section{References}

[1] Alcalde-Unzu, J. and M. Vorsatz (2011). Measuring Consensus: Concepts, Comparisons, and Properties. In: Consensual Processes STUDFUZZ 267 (Eds: Herrera-Viedma, E., J. García-Lapresta, J. Kacprzyk, M. Fedrizzi, H. Nurmi, and H. Zadrozny), pp. 195-211. Springer, Berlin.

[2] Atkinson, A. (1970). On the Measurement of Inequality. Journal of Economic Theory 2, 244-263.

[3] Baldiga, K. and J. Green (2011). Assent-Maximizing Social Choice. Social Choice and Welfare, forthcoming.

[4] Blackorby, C., W. Bossert and D. Donaldson (2005). Population Issues in Social Choice Theory, Welfare Economics, and Ethics. Cambridge University Press.

[5] Bosch, R. (2006). Characterizations of Voting Rules and Consensus Measures. Ph.D. Dissertation, Tilburg University.

[6] Cowell, F. (2008). Measuring Inequality. LSE Perspectives in Economic Analysis, Oxford University Press.

[7] Cook, W. and L. Seiford (1978). Priority Ranking and Consensus Formation. Management Science 24, 1721-1732.

[8] Cook, W. and L. Seiford (1982). On the Borda-Kendall Consensus Method for Priority Ranking Problems. Management Science 28, 621-637.

[9] Duclos, J., J. Esteban, and D. Ray (2006). Polarization: Concepts, Measurement, Estimation. Econometrica 74, 1737-1772.

[10] Echenique, F. and R. Fryer (2007). A Measure of Segregation Based on Social Interactions. Quarterly Journal of Economics 122, 1241-285. 
[11] Eckert, D., C. Klamler, J. Mitloehner, and C. Schloetterer (2006). A DistanceBased Comparison of Basic Voting Rules. Central European Journal of Operations Research 14, 377-386.

[12] Eisenberg, J. (2007). Group Cohesiveness. In: Encyclopedia of Social Psychology (eds. F. Baumeister and K. Vohs), pp. 386-388. Thousands Oaks, Sage.

[13] Elkind, E., P. Faliszewski, and A. Slinko (2010). On the Roles of Distances in Defining Voting Rules. Proceedings of the 9'th International Conference on Autonomous Agents and Multiagent Systems.

[14] Esteban, J. and D. Ray (1994). On the Measurement of Polarization. Econometrica 62, 819-852.

[15] García-Lapresta, J. and D. Pérez-Román (2011). Measuring Consensus in Weak Order. In: Consensual Processes, STUDFUZZ 267 (Eds: HerreraViedma, E., J. García-Lapresta, J. Kacprzyk, M. Fedrizzi, H. Nurmi, and H. Zadrozny), pp. 213-234. Springer, Berlin.

[16] Gini, C. (1921). Measurement of Inequality and Incomes. Economic Journal $31,124-126$.

[17] Hays, W. (1960). A Note on Average Tau as a Measure of Concordance. Journal of the American Statistical Association 55, 331-341.

[18] Hutchens, R. (2004). One Measure of Segregation. International Economic Review 45, 555-578.

[19] Kendall, M. (1962). Rank Correlation Methods, 3rd edition, Hafner Publishing Company, New York.

[20] Kemeny, J. (1959). Mathematics Without Numbers. Daedalus 88, 577-591.

[21] Klamler, C. (2008). A Distance Measure for Choice Functions. Social Choice and Welfare 30, 419-425, 2008.

[22] Kranich, L. (1996). Equitable Opportunities: An Axiomatic Approach. Journal of Economic Theory 71, 131-147.

[23] Meskanen, T. and H. Nurmi (2008). Closeness Counts in Social Choice. In: Power, Freedom, and Voting (Eds: Braham, M. and F. Steffen), pp. 289- 306. Springer, Berlin.

[24] Roemer, J. (1998). Equality of Opportunity. Harvard University Press. 


\section{Appendix}

\section{Proof of Theorem 1}

It is straightforward to show that the family of cohesiveness measures defined in the theorem satisfies the properties. Therefore, we consider from now on the other implication. Take any cohesiveness measure $M$ that satisfies AN, IND, NEU, and MON. First, we show that for all groups $N \subset \mathbb{N}, M^{N}$ is a function of $d\left(P_{N}\right)$; that is, we are going to prove that for any two ranking profiles $P_{N}, P_{N}^{\prime} \in \mathcal{P}^{N}$ such that $d\left(P_{N}\right)=d\left(P_{N}^{\prime}\right), M\left(P_{N}\right)=M\left(P_{N}^{\prime}\right)$.

Take any group $N \subset \mathbb{N}$ and any two ranking profiles $P_{N}, P_{N}^{\prime} \in \mathcal{P}^{N}$ with the property that $d\left(P_{N}\right)=d\left(P_{N}^{\prime}\right)$. Now, consider any unanimous ranking profile $\bar{P}_{N} \in$ $\mathcal{P}^{N}$ (i.e., for all $i, j \in N, \bar{P}_{i}=\bar{P}_{j}$ ) and observe that it is possible to arrive from $P_{N}$ (and $P_{N}^{\prime}$, respectively) to $\bar{P}_{N}$ by means of successive changes of contiguous pairs of objects. Then, given any pair of distinct objects $\{x, y\} \in \bar{X}$, suppose without loss of generality that $\#\left(x P_{N} y\right) \geq \#\left(y P_{N} x\right)$. Now, there are two possibilities: $x \bar{P}_{i} y$ for all $i \in N$ or $y \bar{P}_{i} x$ for all $i \in N$.

1. If $x \bar{P}_{i} y$ for all $i \in N$, in the process of arriving from $P_{N}$ to $\bar{P}_{N}$, the contiguous pair of objects $(y, x)$ has to be changed exactly $\#\left(y P_{N} x\right)$-times to $(x, y)$. Consider first the particular case when $P_{N}$ is such that all individuals agree on how to order all pairs $\{w, z\}$ different from $\{x, y\}$. It follows from MON that each of these changes raises the cohesiveness. Then, by IND, each $x P_{i} y-$ change raises the cohesiveness also if there is no unanimity in the rankings for pairs distinct from $\{x, y\}$. By IND and AN, each incremental only depends on the number of individuals that rank $x$ over $y$ in the profile at which this change is applied. Formally, denote by $t_{j}(x, y)$ the incremental in the cohesiveness when a contiguous pair $(y, x)$ is changed to $(x, y)$ and there were $j$ individuals that rank $x$ over $y$. Also by NEU, $t_{j}(x, y)=t_{j}(z, w) \equiv t_{j}$ for all ordered pairs of objects $(x, y),(z, w)$. Observe that the total incremental in the cohesiveness

from the changes of the contiguous pair $(y, x)$ to $(x, y)$ is $T_{x, y}=\sum_{j=\#\left(x P_{N} y\right)}^{n-1} t_{j}$.

2. On the other hand, if $y \bar{P}_{i} x$ for all $i \in N$, in the process of arriving from 
$P_{N}$ to $\bar{P}_{N}$, the total incremental in the cohesiveness from the changes of the contiguous pair $(x, y)$ to $(y, x)$ equals $T_{y, x}=\sum_{j=\#\left(y P_{N} x\right)}^{n-1} t_{j}$. By IND and NEU, $t_{j}+t_{n-1-j}=0$ for all $j \neq \frac{n-1}{2}$ and, by AN and IND, $t_{\frac{n-1}{2}}=0$ when $n$ is odd. Consequently, $T_{y, x}=T_{x, y}$, which states that the total incremental in the cohesiveness for the changes in the distinct pair of objects $\{x, y\} \in \bar{X}$ in the process of arriving from $P_{N}$ to $\bar{P}_{N}$ is independent on which of the two objects is preferred at any $\bar{P}_{i}$.

Since $d\left(P_{N}\right)=d\left(P_{N}^{\prime}\right)$ by assumption, there exists a bijection $\phi: \bar{X} \rightarrow \bar{X}$ such that $n_{\phi(\{x, y\})}\left(P_{N}^{\prime}\right)=n_{x, y}\left(P_{N}\right)$. We can thus conclude that there exists a pair of objects $\phi(\{x, y\})$ such that all changes of this pair in the process of arriving at $\bar{P}_{N}$ from $P_{N}^{\prime}$ raises the cohesiveness in the same quantity as changes of the pair of objects $\{x, y\}$ in the process of arriving at $\bar{P}_{N}$ from $P_{N}$. Repeating this process for all elements of $\bar{X}$, one can conclude that $M\left(\bar{P}_{N}\right)-M\left(P_{N}^{\prime}\right)=M\left(\bar{P}_{N}\right)-M\left(P_{N}\right)$. Hence, $M\left(P_{N}^{\prime}\right)=M\left(P_{N}\right)$.

It remains to be shown that $M^{N}$ is a linear and additive function of $d\left(P_{N}\right)$. To do that, observe that MON and IND imply some restrictions on $M^{N}$ depending on the values of $d\left(P_{N}\right)$. In particular, MON implies that for all ranking profiles $P_{N}, P_{N}^{\prime} \in \mathcal{P}^{N}$ such that $d_{n}\left(P_{N}^{\prime}\right) \geq d_{n}\left(P_{N}\right)=1-\frac{2}{k(k-1)}$, and for some $i \leq n-2$ $d_{i}\left(P_{N}^{\prime}\right)=d_{i}\left(P_{N}\right)-\frac{2}{k(k-1)}$ and $d_{i+2}\left(P_{N}^{\prime}\right)=d_{i+2}\left(P_{N}\right)+\frac{2}{k(k-1)}, M\left(P_{N}^{\prime}\right)>M\left(P_{N}\right)$. This implication is called Condition 1 from now on. Furthermore, IND implies that for all ranking profiles $P_{N}, P_{N}^{\prime}, \bar{P}_{N}, \bar{P}_{N}^{\prime} \in \mathcal{P}^{N}$ such that for some $i \leq n-2$, $d_{i}\left(P_{N}^{\prime}\right)=d_{i}\left(P_{N}\right)-\frac{2}{k(k-1)}, d_{i+2}\left(P_{N}^{\prime}\right)=d_{i+2}\left(P_{N}\right)+\frac{2}{k(k-1)}, d_{i}\left(\bar{P}_{N}^{\prime}\right)=d_{i}\left(\bar{P}_{N}\right)-$ $\frac{2}{k(k-1)}, d_{i+2}\left(\bar{P}_{N}^{\prime}\right)=d_{i+2}\left(\bar{P}_{N}\right)+\frac{2}{k(k-1)}, d_{j}\left(P_{N}^{\prime}\right)=d_{j}\left(P_{N}\right)$ and $d_{j}\left(\bar{P}_{N}^{\prime}\right)=d_{j}\left(\bar{P}_{N}\right)$ for all $j \neq\{i, i+2\}, M\left(P_{N}^{\prime}\right)-M\left(P_{N}\right)=M\left(\bar{P}_{N}^{\prime}\right)-M\left(\bar{P}_{N}\right)$. We refer to this implication as Condition 2. It is easy to see that, in the presence of Condition 2, Condition 1 can be strengthened in the following way: for all ranking profiles $P_{N}, P_{N}^{\prime} \in \mathcal{P}^{N}$ such that for some $i \leq n-2, d_{i}\left(P_{N}^{\prime}\right)=d_{i}\left(P_{N}\right)-\frac{2}{k(k-1)}, d_{i+2}\left(P_{N}^{\prime}\right)=d_{i+2}\left(P_{N}\right)+\frac{2}{k(k-1)}$ and $d_{j}\left(P_{N}^{\prime}\right)=d_{j}\left(P_{N}\right)$ for all $j \notin\{i, i+2\}, M\left(P_{N}^{\prime}\right)>M\left(P_{N}\right)$. This implication is denoted Condition 3. Next, we proceed by induction separating the proof depending on whether the size of the group is even or odd. 
1. Suppose that $n$ is even. It follows directly from Condition 3 that $M^{N}$ would attain its minimum at any ranking profile $P_{N} \in \mathcal{P}^{N}$ such that $d_{0}\left(P_{N}\right)=1$ and $d_{j}\left(P_{N}\right)=0$ for all $j \neq 0$. It is easy to see that this type of ranking profile exists. An example is a ranking profile such that $\frac{n}{2}$ individuals have any ranking $P_{i} \in \mathcal{P}$ and $\frac{n}{2}$ individuals have the ranking $P_{i}^{O P}$. Without loss of generality, let the cohesiveness at these profiles be $a_{1}^{N}$. Consider now any profile $P_{N}^{\prime}$ such that $d_{i}\left(P_{N}^{\prime}\right)=0$ for all $i>2$. Then, by Condition 2, $M\left(P_{N}^{\prime}\right)=a_{1}^{N}+d_{2}\left(P_{N}^{\prime}\right) \cdot \frac{k(k-1)}{2} \cdot t_{\frac{n}{2}}$. Fix $a_{2}^{N}=a_{1}^{N}+t_{\frac{n}{2}} \cdot \frac{k(k-1)}{2}$.

Suppose now that for any ranking profile $P_{N}^{\prime \prime}$ such that $d_{i}\left(P_{N}^{\prime \prime}\right)=0$ for all $i>q$, with $q$ being an even number from $\{4, \ldots, n-2\}, M\left(P_{N}^{\prime \prime}\right)=a_{1}^{N}+d_{2}\left(P_{N}^{\prime \prime}\right) \cdot t_{\frac{n}{2}}$. $\frac{k(k-1)}{2}+\ldots+d_{q}\left(P_{N}^{\prime \prime}\right) \cdot t_{\frac{n+q-2}{2}} \cdot \frac{k(k-1)}{2}$ and that $a_{\frac{q}{2}+1}^{N}=a_{\frac{q}{2}}^{N}+t_{\frac{n+q-2}{2}} \cdot \frac{k(k-1)}{2}$. Next, consider any ranking profile $\tilde{P}_{N}$ such that $d_{i}\left(\tilde{P}_{N}\right)=0$ for all $i>q+2$. Then, by Condition 2 , we have that $M\left(\tilde{P}_{N}\right)=a_{1}^{N}+d_{2}\left(\tilde{P}_{N}\right) \cdot t_{\frac{n}{2}} \cdot \frac{k(k-1)}{2}+\ldots+d_{q}\left(\tilde{P}_{N}\right)$. $t_{\frac{n+q-2}{2}} \frac{k(k-1)}{2}+d_{q+2}\left(\tilde{P}_{N}\right) \cdot t_{\frac{n+q}{2}} \cdot \frac{k(k-1)}{2}$. Define $a_{\frac{q}{2}+2}^{N}=a_{\frac{q}{2}+1}^{N}+t_{\frac{n+q}{2}} \cdot \frac{k(k-1)}{2}$. Hence, the vector $a^{N}$ is completely defined and it is easy to see that for any arbitrary ranking profile $P_{N} \in \mathcal{P}^{N}, M\left(P_{N}\right)=a^{N} \cdot d\left(P_{N}\right)$.

2. Suppose that $n$ is odd. It follows directly from Condition 3 that $M^{N}$ would attain its minimum at any ranking profile $P_{N} \in \mathcal{P}^{N}$ such that $d_{1}\left(P_{N}\right)=1$ and $d_{i}\left(P_{N}\right)=0$ for all $i \neq 1$. It is easy to see that this type of ranking profile exists. An example is a ranking profile such that $\frac{n-1}{2}$ individuals have any ranking $P_{i} \in \mathcal{P}$ and $\frac{n+1}{2}$ individuals have the ranking $P_{i}^{O P}$. Without loss of generality, let the cohesiveness at these profiles be $a_{1}^{N}$. Consider now any profile $P_{N}^{\prime}$ such that $d_{i}\left(P_{N}^{\prime}\right)=0$ for all $i>3$. Then, by Condition 2 , $M\left(P_{N}^{\prime}\right)=a_{1}^{N}+d_{3}\left(P_{N}^{\prime}\right) \cdot t_{\frac{n+1}{2}} \cdot \frac{k(k-1)}{2}$. Fix $a_{2}^{N}=a_{1}^{N}+t_{\frac{n+1}{2}} \cdot \frac{k(k-1)}{2}$.

Suppose now that for any ranking profile $P_{N}^{\prime \prime}$ such that $d_{i}\left(P_{N}^{\prime \prime}\right)=0$ for all $i>q$, with $q$ being an odd number from $\{5, \ldots, n-2\}, M\left(P_{N}^{\prime \prime}\right)=a_{1}^{N}+d_{3}\left(P_{N}^{\prime \prime}\right) \cdot t_{\frac{n+1}{2}}$. $\frac{k(k-1)}{2}+\ldots+d_{q}\left(P_{N}^{\prime \prime}\right) \cdot t_{\frac{n+q-2}{2}} \cdot \frac{k(k-1)}{2}$ and that $a_{\frac{q+1}{2}}^{N}=a_{\frac{q-1}{2}}^{N}+t_{\frac{n+q-2}{2}} \cdot \frac{k(k-1)}{2}$. Next, consider any ranking profile $\tilde{P}_{N}$ such that $d_{i}\left(\tilde{P}_{N}\right)=0$ for all $i>q+2$. Then, by Condition 2 , we have that $M\left(\tilde{P}_{N}\right)=a_{1}^{N}+d_{3}\left(\tilde{P}_{N}\right) \cdot t_{\frac{n+1}{2}} \cdot \frac{k(k-1)}{2}+\ldots+$ $d_{q}\left(\tilde{P}_{N}\right) \cdot t_{\frac{n+q-2}{2}} \cdot \frac{k(k-1)}{2}+d_{q+2}\left(\tilde{P}_{N}\right) \cdot t_{\frac{n+q}{2}} \cdot \frac{k(k-1)}{2}$. Let $a_{\frac{q+3}{2}}^{N}=a_{\frac{q+1}{2}}^{N}+t_{\frac{n+q}{2}} \cdot \frac{k(k-1)}{2}$. 
Hence, the vector $a^{N}$ is completely defined and it is easy to see that for any arbitrary ranking profile $P_{N} \in \mathcal{P}^{N}, M\left(P_{N}\right)=a^{N} \cdot d\left(P_{N}\right)$.

It follows from AN that $a^{N}=a^{\bar{N}}$ whenever the groups $N$ and $\bar{N}$ are equally sized. Therefore, it can be concluded that for any arbitrary ranking profile $P_{N} \in \mathcal{P}^{N}$, $M\left(P_{N}\right)=a^{n} \cdot d\left(P_{N}\right)$. By Condition $3, t_{i}>0$ for all $i \geq \frac{n}{2}$. This implies that for all $n$ and all $j \in\left\{1,2, \ldots,\left\lfloor\frac{n}{2}\right\rfloor\right\}, a_{j}^{n}<a_{j+1}^{n}$. Given also that the minimal value of $M^{N}$ is attained at all ranking profiles $P_{N} \in \mathcal{P}^{N}$ such that $d_{0}\left(P_{N}\right)=1$ if $n$ is even or $d_{1}\left(P_{N}\right)=1$ if $n$ is odd, and by definition this minimal value has to be nonnegative, we get that $a_{1}^{n} \geq 0$ for all $n$. Finally, by Condition 3 , the maximal value of $M^{N}$ would be attained at all ranking profiles $P_{N} \in \mathcal{P}^{N}$ such that $d_{n}\left(P_{N}\right)=1$. Since these ranking profiles exist (i.e., the unanimous ranking profiles $P_{N}$ such that $P_{i}=P_{j}$ for all $\left.i, j \in N\right)$ and, by definition, the maximal value of $M^{N}$ cannot be greater than 1 , the restriction $a_{\left\lfloor\frac{n}{2}\right\rfloor+1}^{n} \leq 1$ has to be satisfied.

\section{Proof of Theorem 2}

It is straightforward to show that the family of cohesiveness measures defined in the theorem satisfies the properties. Therefore, we consider from now on the other implication. Take any cohesiveness measure $M$ that satisfies IND, NEU, MON, REP, FR, and CON. By Theorem 1, $M \in \Omega$. In the following, we investigate the additional restrictions REP, FR, and CON impose on the set of vectors $a \equiv\left\{a^{n}\right\}_{n \geq 2}$. First, we establish six preliminary claims that will help us to develop the proof later on.

Claim 1 If $n$ is odd, $a_{i}^{n}=a_{2 i}^{2 n}$ for all $i \in\left\{1, \ldots,\left\lfloor\frac{n}{2}\right\rfloor+1\right\}$.

Proof: $\quad$ Take any odd $n$, any $i \in\left\{1, \ldots,\left\lfloor\frac{n}{2}\right\rfloor+1\right\}$, and any ranking profile $P_{N} \in \mathcal{P}^{N}$ such that $d_{2 i-1}\left(P_{N}\right)=1$ and $d_{j}\left(P_{N}\right)=0$ for all $j \neq 2 i-1$. Such a ranking profile always exists. For example, it is a ranking profile in which $\frac{n-1}{2}+i$ individuals have a ranking $P_{i}$ and $\frac{n+1}{2}-i$ have the ranking $P_{i}^{O P}$. Then, by Theorem 1 , we have that $M\left(P_{N}\right)=a_{i}^{n}$. Consider now a group $N^{\prime}$ of size $2 n$ and a ranking profile $P_{N^{\prime}}^{\prime}$ such that it is the union of 2 isomorphic and disjoint copies of $P_{N}$. Then, by definition, 
$d_{4 i-2}\left(P_{N^{\prime}}^{\prime}\right)=1$ and $d_{j}\left(P_{N^{\prime}}^{\prime}\right)=0$ for all $j \neq 4 i-2$. Then, by Theorem 1 , we have that $M\left(P_{N^{\prime}}^{\prime}\right)=a_{2 i}^{2 n}$. By REP, $M\left(P_{N}\right)=M\left(P_{N^{\prime}}^{\prime}\right)$. Therefore, $a_{i}^{n}=a_{2 i}^{2 n}$.

Claim 2 If $n$ is even, $a_{i}^{n}=a_{2 i-1}^{2 n}$ for all $i \in\left\{1, \ldots,\left\lfloor\frac{n}{2}\right\rfloor+1\right\}$.

Proof: Consider any even $n$ and any $i \in\left\{1, \ldots, \frac{n}{2}+1\right\}$. Take any ranking profile $P_{N} \in \mathcal{P}^{N}$ such that $d_{2 i-2}\left(P_{N}\right)=1$ and $d_{j}\left(P_{N}\right)=0$ for all $j \neq 2 i-2$. Such a ranking profile always exists. For example, it is a ranking profile in which $\frac{n}{2}+i-1$ individuals have a ranking $P_{i}$ and $\frac{n}{2}-i+1$ have the ranking $P_{i}^{O P}$. Then, by Theorem 1, we have that $M\left(P_{N}\right)=a_{i}^{n}$. Consider now a group $N^{\prime}$ of size $2 n$ and a ranking profile $P_{N^{\prime}}^{\prime}$ such that it is the union of 2 isomorphic and disjoint copies of $P_{N}$. Then, by definition, $d_{4 i-4}\left(P_{N^{\prime}}^{\prime}\right)=1$ and $d_{j}\left(P_{N^{\prime}}^{\prime}\right)=0$ for all $j \neq 4 i-4$. Then, by Theorem 1 , we have that $M\left(P_{N^{\prime}}^{\prime}\right)=a_{2 i-1}^{2 n}$. By REP, $M\left(P_{N}\right)=M\left(P_{N^{\prime}}^{\prime}\right)$. Therefore, $a_{i}^{n}=a_{2 i-1}^{2 n}$.

Claim 3 If $n$ is odd, $a_{1}^{n}>0$.

Proof: Suppose otherwise. Then, there is some odd $n$ such that $a_{1}^{n}=0$. Then, by Claim $1, a_{2}^{2 n}=0$. Since $a_{j}^{t}<a_{j+1}^{t}$ for all $t$ and all $j \in\left\{1, \ldots,\left\lfloor\frac{t}{2}\right\rfloor\right\}$ by Theorem 1 , it must be the case that $a_{1}^{2 n}<0$. But this is not possible given that $a_{1}^{t} \geq 0$ for all $t$ by Theorem 1 .

Claim 4 If $n$ is even, $a_{1}^{n}=0$.

Proof: Consider any even $n$. By FR, there is some group $C$ of size $c$ and some ranking profile $P_{C}^{\prime} \in \mathcal{P}^{C}$ such that $M\left(P_{C}^{\prime}\right)=0$. It follows from Theorem 1 that the minimal value of $M^{C}$ is $a_{1}^{c}$. Then, it follows from Claim 3 that $c$ is an even number. By definition, $P_{C}^{\prime}$ should be one ranking profile with the minimal value of $M^{C}$. By Theorem 1 , this minimal value is attained in ranking profiles $P_{C}^{\prime \prime}$ satisfying that $d_{0}\left(P_{C}^{\prime \prime}\right)=1$, which always exist. An example is $P_{C}^{\prime \prime}$ such that $\frac{c}{2}$ individuals have the ranking $P_{i}^{\prime \prime}$ and the other $\frac{c}{2}$ individuals have the ranking $\left(P_{i}^{\prime \prime}\right)^{O P}$. By Theorem 1, $M\left(P_{C}^{\prime \prime}\right)=M\left(P_{C}^{\prime}\right)=0$.

Take any pair of individuals $\{i, j\} \subseteq C$ such that $P_{i}^{\prime \prime} \neq P_{j}^{\prime \prime}$. By REP, $M\left(P_{\{i, j\}}^{\prime \prime}\right)=$ $M\left(P_{C}^{\prime \prime}\right)=0$. Since $d_{0}\left(P_{\{i, j\}}^{\prime \prime}\right)=1$ and $d_{2}\left(P_{\{i, j\}}^{\prime \prime}\right)=0$, we know that $M\left(P_{\{i, j\}}^{\prime \prime}\right)=a_{1}^{2}$ 
by Theorem 1 . Hence, $a_{1}^{2}=0$. Now, consider the ranking profile $P_{N}$ that consists of the union of $\frac{n}{2}$ isomorphic and disjoint copies of $P_{\{i, j\}}^{\prime \prime}$. Then, $d_{0}\left(P_{N}\right)=1$ and $d_{j}\left(P_{N}\right)=0$ for all $j \neq 0$. By Theorem 1 , we have that $M\left(P_{N}\right)=a_{1}^{n}$. By REP, we have that $M\left(P_{N}\right)=M\left(P_{\{i, j\}}^{\prime \prime}\right)=0$. Therefore, $a_{1}^{n}=0$.

Claim 5 For all $n, a_{\left\lfloor\frac{n}{2}\right\rfloor+1}^{n}=1$.

Proof: Consider any $n$. Observe first that, by $\mathrm{FR}$, there exists a group $C$ of size $c$ and a ranking profile $P_{C}^{\prime} \in \mathcal{P}^{C}$ for which the cohesiveness is 1 . By definition, $P_{C}^{\prime}$ should be one ranking profile with the maximal value of $M^{C}$. By Theorem 1, this maximal value is attained in ranking profiles $P_{C}^{\prime \prime}$ satisfying that $d_{c}\left(P_{C}^{\prime \prime}\right)=1$ and $d_{i}\left(P_{C}^{\prime \prime}\right)=0$ for all $i \neq c$, which always exist. An example is $P_{C}^{\prime \prime}$ such that $P_{i}^{\prime \prime}=P_{j}^{\prime \prime}$ for all $i, j \in C$. By Theorem $1, M\left(P_{C}^{\prime}\right)=M\left(P_{C}^{\prime \prime}\right)=a_{\left\lfloor\frac{c}{2}\right\rfloor+1}^{c}=1$. Consider now the profile $\bar{P}_{B}$ consisting in the union of $n$ isomorphic and disjoint copies of $P_{C}^{\prime}$. Then, $d_{n c}\left(\bar{P}_{B}\right)=1$ and $d_{i}\left(\bar{P}_{B}\right)=0$ for all $i \neq n c$. By REP, $M\left(\bar{P}_{B}\right)=M\left(P_{C}^{\prime}\right)=1$. Therefore, $a_{\left\lfloor\frac{n c}{2}\right\rfloor+1}^{n c}=1$. Consider the profile $P_{N}$ of size $n$ such that all individuals $i \in N$ have the ranking $P_{i}^{\prime \prime}$. Note that $d_{n}\left(P_{N}\right)=1$ and $d_{i}\left(P_{N}\right)=0$ for all $i \neq n$. Then, $M\left(P_{N}\right)=a_{\left\lfloor\frac{n}{2}\right\rfloor+1}^{n}$ and $\bar{P}_{B}$ consists in the union of $c$ isomorphic and disjoint copies of $P_{N}$. Then, by REP, $M\left(P_{N}\right)=M\left(\bar{P}_{B}\right)=1$. Therefore, $a_{\left\lfloor\frac{n}{2}\right\rfloor+1}^{n}=1$.

Claim 6 If $n$ is a multiple of $4, a_{2}^{4}=a_{\frac{n}{4}+1}^{n}$.

Proof: Consider any $n$ multiple of 4 , a group $C$ of size 4 and a ranking profile $P_{C}^{\prime} \in \mathcal{P}^{C}$ such that $d_{2}\left(P_{C}^{\prime}\right)=1$ and $d_{j}\left(P_{C}^{\prime}\right)=0$ for all $j \neq 2$. Then, $M\left(P_{C}^{\prime}\right)=a_{2}^{4}$. Consider now the ranking profile $P_{N}$ of size $n$ consisting in the union of $\frac{n}{4}$ isomorphic and disjoint copies of $P_{C}^{\prime}$. Note that $d_{\frac{n}{2}}\left(P_{N}\right)=1$ and $d_{j}\left(P_{N}\right)=0$ for all $j \neq \frac{n}{2}$. Then, $M\left(P_{N}\right)=a_{\frac{n}{4}+1}^{n}$. By REP, $M\left(P_{N}\right)=M\left(P_{C}^{\prime}\right)$. Therefore, $a_{2}^{4}=a_{\frac{n}{4}+1}^{n}$.

To find the exact description of the set of vectors $a$, we divide the proof into two parts, depending on whether the size of the group $N$ is even or odd.

1. Suppose that $n$ is even. We know from Claims 4 and 5 that $a_{1}^{n}=0$ and $a_{\frac{n}{2}+1}^{n}=1$. This concludes the proof for the case $n=2$. Therefore, suppose next that $n \geq 4$. If $n=4$, the unique value not determined by the 
axioms is $a_{2}^{4}$. Define $\gamma \equiv a_{2}^{4}$ and observe that, for the moment, by Theorem 1 , $\gamma \in(0,1)$. If $n \geq 6$, it follows from CON and IND that for all $i \in\left\{1, \ldots, \frac{n}{2}-\right.$ $2\},\left(\left(a_{i+2}^{n}-a_{i+1}^{n}\right)-\left(a_{i+1}^{n}-a_{i}^{n}\right)\right)=\left(\left(a_{i+3}^{n}-a_{i+2}^{n}\right)-\left(a_{i+2}^{n}-a_{i+1}^{n}\right)\right)$. Consequently, if we define $\bar{p}(n, \gamma) \equiv a_{2}^{n}$ and $\bar{q}(n, \gamma) \equiv\left(\left(a_{i+2}^{n}-a_{i+1}^{n}\right)-\left(a_{i+1}^{n}-a_{i}^{n}\right)\right)$ for any $i \in\left\{1, \ldots, \frac{n}{2}-2\right\}$, we have that $a_{i}^{n}=(i-1) \bar{p}(n, \gamma)+\frac{(i-2)(i-1)}{2} \bar{q}(n, \gamma)$ for all $i \in\left\{1, \ldots, \frac{n}{2}+1\right\}$.

In case $n$ is a multiple of $4, a_{\frac{n}{4}+1}^{n}=a_{2}^{4}=\gamma$ by Claim 6 . Using this and Claim 5 we obtain the following set of linear equations:

$$
\begin{aligned}
& \frac{n}{2} \bar{p}(n, \gamma)+\frac{\frac{n}{2}\left(\frac{n}{2}-1\right)}{2} \bar{q}(n, \gamma)=1 \\
& \frac{n}{4} \bar{p}(n, \gamma)+\frac{\frac{n}{4}\left(\frac{n}{4}-1\right)}{2} \bar{q}(n, \gamma)=\gamma .
\end{aligned}
$$

Solving for $\bar{p}(n, \gamma)$ and $\bar{q}(n, \gamma)$ yields

$$
\begin{aligned}
\bar{p}(n, \gamma) & =\frac{1}{n^{2}}(16 \gamma+(8 \gamma-2)(n-4)) \\
\bar{q}(n, \gamma) & =\frac{1}{n^{2}}(16-32 \gamma) .
\end{aligned}
$$

Letting $p(n, \gamma)=n^{2} \bar{p}(n, \gamma)$ and $q(n, \gamma)=n^{2} \bar{q}(n, \gamma)$, one can easily check that if $n$ is a multiple of 4 , then for all $i \in\left\{1, \ldots, \frac{n}{2}+1\right\}$.

$$
a_{i}^{n}=\frac{i-1}{2 n^{2}}(2 p(n, \gamma)+(i-2) q(n, \gamma)) .
$$

Then, we have that

$$
a_{i}^{n}=\frac{i-1}{2 n^{2}}[2(16 \gamma+(8 \gamma-2)(n-4))+(i-2)(16-32 \gamma)] .
$$

Slightly tedious but straightforward manipulations of this equation imply that

$$
a_{i}^{n}=\frac{2(i-1)}{n^{2}}[n(4 \gamma-1)+2(i-1)(2-4 \gamma)],
$$

which can be easily rewritten in the desired form:

$$
a_{i}^{n}=\frac{2(i-1)}{n^{2}}[4(\gamma-0.25) \cdot n+(1-4(\gamma-0.25)) \cdot 2(i-1)] .
$$

For all other even values of $n$, observe that $a_{i}^{n}=a_{2 i-1}^{2 n}$ by Claim 2. Then, given that $2 n$ is a multiple of 4 , we have that for all $i \in\left\{1, \ldots, \frac{n}{2}+1\right\}$,

$$
a_{i}^{n}=\frac{2(2 i-2)}{4 n^{2}}[4(\gamma-0.25) \cdot 2 n+(1-4(\gamma-0.25)) \cdot 2(2 i-2)] .
$$

It can be easily seen that this is the desired result. 
2. Suppose now that $n$ is odd. We know from Claim 1 that $a_{i}^{n}=a_{2 i}^{2 n}$. Consequently, we have that for all $i \in\left\{1, \ldots,\left\lfloor\frac{n}{2}\right\rfloor+1\right\}$,

$$
a_{i}^{n}=\frac{2(2 i-1)}{4 n^{2}}[4(\gamma-0.25) \cdot 2 n+(1-4(\gamma-0.25)) \cdot 2(2 i-1)] .
$$

It can be easily seen that this is the desired result.

Finally, it remains to be shown that $\gamma \in\left[\frac{1}{4}, \frac{3}{4}\right]$. We know from Theorem 1 that for all $n$ and all $i \in\left\{2, \ldots,\left\lfloor\frac{n}{2}\right\rfloor+1\right\}$, it is necessary that $a_{i}^{n}-a_{i-1}^{n}>0$. To guarantee it, we can focus on the cases in which $n$ is even and strictly greater than $2 .^{5} \mathrm{We}$ have then that for all $i \in\left\{2, \ldots, \frac{n}{2}+1\right\}, a_{i}^{n}-a_{i-1}^{n}=\bar{p}(n, \gamma)+(i-2) \bar{q}(n, \gamma)$. Since this equation reduces to $a_{2}^{n}-a_{1}^{n}=\bar{p}(n, \gamma)$ for $i=2$, it is necessary that $p(n, \gamma)>0$ for all $n$ even and strictly greater than 2. Consequently, it is required that $16 \gamma+(8 \gamma-2)(n-4)>0$ for all $n$ even and strictly greater than 2 . It is easy to see that if this inequality holds for $n \rightarrow \infty$, it also holds for all $n$ even and strictly greater than 2. For $n \rightarrow \infty$ this equation reduces to $8 \gamma-2 \geq 0$, which is equivalent to $\gamma \geq \frac{1}{4}$.

To establish that $a_{i}^{n}-a_{i-1}^{n}>0$ for all $i \in\left\{3, \ldots, \frac{n}{2}+1\right\}$, we only have to show that $a_{\frac{n}{2}+1}^{n}-a_{\frac{n}{2}}^{n}=\bar{p}(n, \gamma)+\left(\frac{n}{2}-1\right) \bar{q}(n, \gamma)>0$. This is because $\bar{p}(n, \gamma)>0$ for $\gamma \geq \frac{1}{4}$ and for any negative $\bar{q}(n, \gamma), a_{i}^{n}-a_{i-1}^{n}$ is minimized for $i=\frac{n}{2}+1$. Using some algebra, it can be verified that the necessary condition is $n(6-8 \gamma)+8(2 \gamma-1)>0$. Again, it is sufficient to check the condition for $n \rightarrow \infty$. For $n \rightarrow \infty$ this equation reduces to $6-8 \gamma \geq 0$, which is equivalent to $\gamma \leq \frac{3}{4}$.

\section{Proof of Theorem 3}

It is straightforward to show that $\hat{\tau}$ satisfies the set of axioms. To show the other implication, consider any cohesiveness measure $M$ that satisfies REP, NEU, PROP, MON, and FR. Since IND and CON are implied by the other five properties, Theorem 2 applies and we only have to determine $\gamma=a_{2}^{4}$. Since it follows from Theorem 2 that $a_{1}^{4}=0$ and $a_{3}^{4}=1$, we can apply PROP to obtain that $\frac{\gamma}{1-\gamma}=\frac{1}{3}$. This equation solves for $\gamma=0.25$.

\footnotetext{
${ }^{5}$ To see why, suppose that $n$ is odd and that $a_{i}^{n}<a_{i-1}^{n}$ for some $i \in\left\{1, \ldots,\left\lfloor\frac{n}{2}\right\rfloor+1\right\}$. Then, by Claim $2, a_{2 i}^{2 n}<a_{2 i-2}^{2 n}$. Hence, the condition is also violated for some even $n$. For the special case when $n=2$, we already know that $a_{2}^{2}=1>0=a_{1}^{2}$.
} 


\section{Proof of Theorem 4}

It is straightforward to show that $\bar{\sigma}$ satisfies the set of axioms. Similarly, some basic calculus can show that $\bar{\sigma}$ can be formulated as stated in points 2 and 3 . Then, we have to show only that the set of axioms of point 1 imply that the cohesiveness measure is exactly the measure of $\Gamma$ with $\gamma=\frac{1}{2}$. To show it, consider any cohesiveness measure $M$ that satisfies REP, SIND, NEU, MON, and FR. Since IND and CON are implied by the other five properties, Theorem 2 applies and we only have to determine $\gamma=a_{2}^{4}$. Since it follows from Theorem 2 that $a_{1}^{4}=0$ and $a_{3}^{4}=1$, we can apply SIND to obtain that $\gamma-0=1-\gamma$. Hence, $\gamma=0.5$.

\section{Independence in Theorem 1}

We show by means of four examples that the properties in Theorem 1 are independent.

Anonymity: For every group $N \subset \mathbb{N}$, assign to each group of individuals $A \subseteq N$ a non-negative number $p_{N}(A)$ such that $p_{N}(A)=0$ if $\# A \leq \frac{n}{2}, p_{N}(A)>p_{N}(B)$ if $\# A>\# B$ and $\# A>\frac{n}{2}, p_{N}(N)=\frac{2}{k(k-1)}$, and $p_{N^{\prime}}(A) \neq p_{N^{\prime}}(B)$ for some group $N^{\prime} \subset \mathbb{N}$ and some $A, B \subset N^{\prime}$ such that $A \neq B$ and $\# A=\# B$. Given a ranking profile $P_{N}$ and a pair of objects $\{x, y\} \in \bar{X}$, denote the value assigned to the maximal set of individuals who prefer the object that is favored by the majority as $s_{x, y}\left(P_{N}\right)=p_{N}\left(\left\{A \subseteq N: \#\left(x P_{A} y\right) \geq \#\left(y P_{A} x\right)\right.\right.$ and there is no $B \subseteq$ $N$ s.t. $\left.\left.\#\left(x P_{B} y\right)>\#\left(x P_{A} y\right)\right\}\right)$. Now, let cohesiveness measure $M_{1}$ be such that for all groups $N \in \mathbb{N}$ and all ranking profiles $P_{N}, M_{1}\left(P_{N}\right)=\sum_{\{x, y\} \in \bar{X}} s_{x, y}\left(P_{N}\right)$. This cohesiveness measure satisfies NEU, IND, and MON. The following example shows that it is not anonymous. Let $N=\{1,2,3\}$ and $X=\{x, y\}$. Suppose that the ranking profiles $P_{N}$ and $P_{N}^{\prime}$ are such that $x P_{1} y, x P_{2} y, y P_{3} x, y P_{1}^{\prime} x, x P_{2}^{\prime} y$, and $x P_{3}^{\prime} y$. Moreover, let $p_{N}(\{1,2\})=p_{N}(\{1,3\})=\frac{1}{2}$ and $p_{N}(\{2,3\})=\frac{3}{4}$. Then, $M_{1}\left(P_{N}\right)=\frac{1}{2}$ and $M_{1}\left(P_{N}^{\prime}\right)=\frac{3}{4}$. AN would imply that $M_{1}\left(P_{N}\right)=M_{1}\left(P_{N}^{\prime}\right)$.

Neutrality: Let $q: \bar{X} \rightarrow \mathbb{R}_{++}$be a function that assigns to each pair of objects $\{x, y\} \in \bar{X}$ a strictly positive weight $q_{x, y}>0$ in such a way that $q_{x, y} \neq q_{w, z}$ for 
some $\{w, z\} \in(\bar{X} \backslash\{\{x, y\}\})$. Now, let cohesiveness measure $M_{2}$ be such that for all groups $N \in \mathbb{N}$ and all ranking profiles $P_{N}, M_{2}\left(P_{N}\right)=\sum_{\{x, y\} \in \bar{X}} \frac{q_{x, y}}{\sum_{\{w, z\} \in \bar{X}} q_{w, z}} \sigma_{x, y}\left(P_{N}\right)$. This cohesiveness measure satisfies AN, IND, and MON. The following example shows that it is not neutral. Let $N=\{1,2\}$ and $X=\{x, y, z\}$. Suppose that the ranking profiles $P_{N}$ and $P_{N}^{\prime}$ are such that $x P_{1} y P_{1} z, y P_{2} x P_{2} z, z P_{1}^{\prime} y P_{1}^{\prime} x$, and $y P_{2}^{\prime} z P_{2}^{\prime} x$. Moreover, let $q_{x, z}=q_{y, z}=1$ and $q_{x, y}=2$. Then, $M_{2}\left(P_{N}\right)=\frac{2}{4}$ and $M_{2}\left(P_{N}^{\prime}\right)=\frac{3}{4}$. NEU would imply that $M_{2}\left(P_{N}\right)=M_{2}\left(P_{N}^{\prime}\right)$.

Independence: $\quad$ Let $f:\{1, \ldots, k\} \rightarrow \mathbb{R}_{++}$be a function that assigns to each position in a ranking a strictly positive weight such that $f(v) \neq f(w)$ for some $v, w \in\{1, \ldots, k\}$. Then, for each group $N \subset \mathbb{N}$, define the function $F_{N}: X \times$ $\mathcal{P}^{N} \rightarrow \mathbb{R}_{++}$as $F_{N}\left(x, P_{N}\right)=\sum_{i \in N} f\left(\#\left\{y \in X: \neg y P_{i} x\right\}\right)$. Let the cohesiveness measure $M_{3}$ be such that for all groups $N \in \mathbb{N}$ and all ranking profiles $P_{N}$, $M_{3}\left(P_{N}\right)=\sum_{\{x, y\} \in \bar{X}} \frac{F_{N}\left(x, P_{N}\right)+F_{N}\left(y, P_{N}\right)}{\sum_{\{w, z\} \in \bar{X}} F_{N}\left(w, P_{N}\right)+F_{N}\left(z, P_{N}\right)} \sigma_{x, y}\left(P_{N}\right)$. This cohesiveness measure satisfies AN, NEU, and MON. The following example shows that it is not independent. Let $N=\{1,2,3\}$ and $X=\{x, y, z\}$. Suppose that the ranking profiles $P_{N}$ and $\bar{P}_{N}$ are such that $x P_{1} y P_{1} z, P_{2}=P_{1}, y P_{3} x P_{3} z, z \bar{P}_{1} x \bar{P}_{1} y, \bar{P}_{2}=\bar{P}_{1}$, and $z \bar{P}_{3} y \bar{P}_{3} x$. Let the ranking profiles $P_{N}^{\prime}$ and $\bar{P}_{N}^{\prime}$ be obtained by performing a $y P_{3} x$-change and a $y \bar{P}_{3} x$-change, respectively. Moreover, let $f(1)=3, f(2)=2$ and $f(3)=1$. Then $M_{3}\left(P_{N}^{\prime}\right)-M_{3}\left(P_{N}\right)=1-\frac{2}{3}=\frac{1}{3}$ and $M_{3}\left(\bar{P}_{N}^{\prime}\right)-M_{3}\left(\bar{P}_{N}\right)=1-\frac{8}{9}=\frac{1}{9}$. IND would imply that $M_{3}\left(P_{N}^{\prime}\right)-M_{3}\left(P_{N}\right)=M_{3}\left(\bar{P}_{N}^{\prime}\right)-M_{3}\left(\bar{P}_{N}\right)$.

Monotonicity: Let the cohesiveness measure $M_{4}$ be such that for all groups $N \in \mathbb{N}$ and all ranking profiles $P_{N}, M_{4}\left(P_{N}\right)=1-\bar{\sigma}\left(P_{N}\right)$. This cohesiveness measure satisfies AN, NEU, and IND. The following example shows that it is not monotonic. Let $N=\{1,2\}$ and $X=\{x, y\}$. Suppose that the ranking profiles $P_{N}$ and $P_{N}^{\prime}$ are such that $x P_{1} y, y P_{2} x, x P_{1}^{\prime} y$, and $x P_{2}^{\prime} y$. Then, $M_{4}\left(P_{N}\right)=1$ and $M_{4}\left(P_{N}^{\prime}\right)=0$. MON would imply that $M_{4}\left(P_{N}^{\prime}\right)>M_{4}\left(P_{N}\right)$. 


\section{Independence in Theorem 2}

We show by means of six examples that the properties in Theorem 2 are independent.

Replication Invariance: As we showed in Section 5.1, the measure $\bar{\tau}$ satisfies NEU, IND, MON, FR and CON, but it does not satisfy REP.

Neutrality: The cohesiveness measure $M_{2}$ satisfies REP, IND, MON, FR, and CON. However, it is not neutral.

Independence: The cohesiveness measure $M_{3}$ satisfies REP, NEU, MON, FR, and CON. However, it is not independent.

Monotonicity: The cohesiveness measure $M_{4}$ satisfies REP, NEU, IND, FR, and CON. However, it is not monotonic.

Full Range: Let the cohesiveness measure $M_{5}$ be such that for all groups $N \in \mathbb{N}$ and all ranking profiles $P_{N}, M_{6}\left(P_{N}\right)=\frac{1}{2} \bar{\sigma}\left(P_{N}\right)$. This cohesiveness measure satisfies REP, NEU, IND, MON, and CON. Since its maximum is $\frac{1}{2}$, it does not satisfy Full Range.

Concordance: For every group $N \subset \mathbb{N}$, denote the vector of weights associated with the measure $\bar{\sigma}$ by $a^{n}(\bar{\sigma})$. Now, let the cohesiveness measure $M_{6} \in \Omega$ be such that for all $n, a_{\left\lfloor\frac{n}{2}\right\rfloor+1}^{n}=1$ and $a_{i}^{n}=\frac{a_{i}^{n}(\bar{\sigma})}{2}$ for all $i \in\left\{1, \ldots,\left\lfloor\frac{n}{2}\right\rfloor\right\}$. This cohesiveness measure satisfies REP, NEU, IND, MON, and FR. The following example shows that it is not concordant. Suppose that $N=\{1,2,3,4,5,6\}$ and $X=\{x, y\}$. Let $P_{N}$ be such that $x P_{1} y, y P_{4} x, P_{1}=P_{2}=P_{3}$, and $P_{4}=P_{5}=P_{6}$. Then, $M_{6}\left(P_{N}\right)=0$. If the ranking profile $P_{N}^{\prime}$ is $(x, y)$-different from $P_{N}$ for 4 , then $M_{6}\left(P_{N}^{\prime}\right)=\frac{1}{6}$. If the ranking profile $P_{N}^{\prime \prime}$ is $(x, y)$-different from $P_{N}^{\prime}$ for individual 5 , then $M_{6}\left(P_{N}^{\prime \prime}\right)=\frac{1}{3}$. Finally, if the ranking profile $P_{N}^{\prime \prime \prime}$ is $(x, y)$-different from $P_{N}^{\prime \prime}$ for individual 6 , then $M_{6}\left(P_{N}^{\prime \prime \prime}\right)=1$. Consequently, $\left(M_{6}\left(P_{N}^{\prime \prime}\right)-M_{6}\left(P_{N}^{\prime}\right)\right)-\left(M_{6}\left(P_{N}^{\prime}\right)-M_{6}\left(P_{N}\right)\right)=\frac{1}{6}-\frac{1}{6}=0$, whereas $\left(M_{6}\left(P_{N}^{\prime \prime \prime}\right)-M_{6}\left(P_{N}^{\prime \prime}\right)\right)-\left(M_{6}\left(P_{N}^{\prime \prime}\right)-M_{6}\left(P_{N}^{\prime}\right)\right)=\frac{2}{3}-\frac{1}{6}=\frac{1}{2}$. CON would imply that 
$\left(M_{6}\left(P_{N}^{\prime \prime}\right)-M_{6}\left(P_{N}^{\prime}\right)\right)-\left(M_{6}\left(P_{N}^{\prime}\right)-M_{6}\left(P_{N}\right)\right)=\left(M_{6}\left(P_{N}^{\prime \prime \prime}\right)-M_{6}\left(P_{N}^{\prime \prime}\right)\right)-\left(M_{6}\left(P_{N}^{\prime \prime}\right)-\right.$ $\left.M_{6}\left(P_{N}^{\prime}\right)\right)$.

\section{Independence in Theorem 3}

We show by means of five examples that the properties in Theorem 3 are independent.

Replication Invariance: The cohesiveness measure $\bar{\tau}$ satisfies NEU, PROP, MON, and FR. However, it does not satisfy REP.

Neutrality: Let $q: \bar{X} \rightarrow \mathbb{R}_{++}$be a function that assigns to each pair of objects $\{x, y\} \in \bar{X}$ a strictly positive weight $q_{x, y}>0$ in such a way that $q_{x, y} \neq q_{w, z}$ for some $\{w, z\} \in(\bar{X} \backslash\{\{x, y\}\})$. Given a group $N \in \mathbb{N}$, a ranking profile $P_{N}$, and two distinct individuals $i, j \in N$, let $w_{i, j}\left(P_{N}\right)=\sum_{\{x, y\} \in \bar{X}} \frac{q_{x, y}}{\sum_{\{w, z\} \in \bar{X}} q_{w, z}} \cdot \tau_{i, j}^{x, y}\left(P_{N}\right)$ be the weighted percentage of pairwise comparisons individual $i$ and $j$ agree upon. Define $\bar{w}\left(P_{N}\right)=\frac{2}{n(n-1)} \sum_{i, j \in N: j \neq i} w_{i, j}\left(P_{N}\right)$ as the average weighted tau. Now, let cohesiveness measure $M_{7}$ be such that for all groups $N \in \mathbb{N}$ and all ranking profiles $P_{N}, M_{7}\left(P_{N}\right)=\frac{\bar{w}\left(P_{N}\right)-\frac{1}{n}}{\frac{n-1}{n}}$ if $n$ is even and $M_{7}\left(P_{N}\right)=\frac{1}{n^{2}}+\frac{\bar{w}\left(P_{N}\right)-\frac{n-1}{2 n}}{\frac{n+1}{2 n}} \frac{n^{2}-1}{n^{2}}$ if $n$ is odd. This cohesiveness measure satisfies REP, PROP, MON, and FR. The following example shows that it is not neutral. Let $N=\{1,2\}$ and $X=\{x, y, z\}$. Suppose that the ranking profiles $P_{N}$ and $P_{N}^{\prime}$ are such that $x P_{1} y P_{1} z, y P_{2} x P_{2} z, z P_{1}^{\prime} y P_{1}^{\prime} x$, and $y P_{2}^{\prime} z P_{2}^{\prime} x$. Moreover, let $q_{x, z}=q_{y, z}=1$ and $q_{x, y}=2$. Then, $M_{7}\left(P_{N}\right)=\frac{2}{4}$ and $M_{7}\left(P_{N}^{\prime}\right)=\frac{3}{4}$. NEU would imply that $M_{7}\left(P_{N}\right)=M_{7}\left(P_{N}^{\prime}\right)$.

Proportionality: Let the cohesiveness measure $M_{8}$ be such that for all groups $N \in \mathbb{N}$ and all ranking profiles $P_{N}, M_{8}\left(P_{N}\right)=\bar{\sigma}\left(P_{N}\right)$. This cohesiveness measure satisfies REP, NEU, MON, and FR. The following example shows that it is not proportional. Let $N=\{1,2,3,4\}$ and $X=\{x, y\}$. Suppose that the ranking profiles $P_{N}$ and $\bar{P}_{N}$ are such that $x P_{1} y, y P_{2} x, P_{3}=P_{1}, P_{4}=P_{2}, \bar{P}_{1}=\bar{P}_{2}=\bar{P}_{3}=P_{1}$, and $\bar{P}_{4}=P_{2}$. Let the ranking profiles $P_{N}^{\prime}$ and $\bar{P}_{N}^{\prime}$ be obtained by performing a $y P_{4} x$-change and a $y \bar{P}_{4} x$-change, respectively. Then, $M_{8}\left(P_{N}^{\prime}\right)-M_{8}\left(P_{N}\right)=\frac{1}{2}-0=\frac{1}{2}$ and $M_{8}\left(\bar{P}_{N}^{\prime}\right)-M_{8}\left(\bar{P}_{N}\right)=1-\frac{1}{2}=\frac{1}{2}$. PROP would imply that $\frac{M_{8}\left(P_{N}^{\prime}\right)-M_{8}\left(P_{N}\right)}{M_{8}\left(\bar{P}_{N}^{\prime}\right)-M_{8}\left(\bar{P}_{N}\right)}=\frac{1}{3}$. 
Monotonicity: Let the cohesiveness measure $M_{9}$ be such that for all groups $N \in \mathbb{N}$ and all ranking profiles $P_{N}, M_{9}\left(P_{N}\right)=1-\hat{\tau}\left(P_{N}\right)$. This cohesiveness measure satisfies REP, NEU, PROP, and FR. The following example shows that it is not monotonic. Let $N=\{1,2\}$ and $X=\{x, y\}$. Suppose that the ranking profiles $P_{N}$ and $P_{N}^{\prime}$ are such that $x P_{1} y, y P_{2} x, x P_{1}^{\prime} y$, and $x P_{2}^{\prime} y$. Then, $M_{9}\left(P_{N}\right)=1$ and $M_{9}\left(P_{N}^{\prime}\right)=0$. MON would imply that $M_{9}\left(P_{N}^{\prime}\right)>M_{9}\left(P_{N}\right)$.

Full Range: Let the cohesiveness measure $M_{10}$ be such that for all groups $N \in$ $\mathbb{N}$ and all ranking profiles $P_{N}, M_{10}\left(P_{N}\right)=\frac{1}{2} \hat{\tau}\left(P_{N}\right)$. This cohesiveness measure satisfies REP, NEU, PROP, and MON. Since its maximum is $\frac{1}{2}$, it does not satisfy FR.

\section{Independence in Theorem 4}

We show by means of five examples that the properties in Theorem 4 are independent.

Replication Invariance: Let the cohesiveness measure $M_{11}$ be such that for all groups $N \in \mathbb{N}$ and all ranking profiles $P_{N}, M_{11}\left(P_{N}\right)=\bar{\sigma}\left(P_{N}\right)$ whenever $n$ is even and $M_{11}\left(P_{N}\right)=\frac{\bar{\sigma}\left(P_{N}\right)-\frac{1}{n}}{1-\frac{1}{n}}$ if $n$ is odd. This cohesiveness measure satisfies NEU, SIND, MON, and FR. The following example shows that it does not satisfy REP. Let $N=\{1,2,3\}, \bar{N}=\{1,2,3,4,5,6\}$, and $X=\{x, y\}$. Let $x P_{1} y, y P_{2} x, P_{3}=P_{4}=$ $P_{6}=P_{1}$, and $P_{5}=P_{2}$. Then, $M_{11}\left(P_{N}\right)=0$ and $M_{11}\left(P_{\bar{N}}\right)=\frac{1}{3}$. REP would imply that $M_{11}\left(P_{\bar{N}}\right)=M_{11}\left(P_{N}\right)$.

Neutrality: The cohesiveness measure $M_{2}$ satisfies REP, SIND, MON, and FR. However, it is not neutral.

Strong Independence: Let the cohesiveness measure $M_{12}$ be such that for all groups $N \in \mathbb{N}$ and all ranking profiles $P_{N}, M_{12}\left(P_{N}\right)=\hat{\tau}\left(P_{N}\right)$. This cohesiveness measure satisfies REP, NEU, MON, and FR. The following example shows that it is not strongly independent. Let $N=\{1,2,3,4\}$ and $X=\{x, y\}$. Suppose that the ranking profiles $P_{N}$ and $\bar{P}_{N}$ are such that $x P_{1} y, y P_{2} x, P_{3}=P_{1}, P_{4}=P_{2}, \bar{P}_{1}=$ 
$\bar{P}_{2}=\bar{P}_{3}=P_{1}$, and $\bar{P}_{4}=P_{2}$. Let the ranking profiles $P_{N}^{\prime}$ and $\bar{P}_{N}^{\prime}$ be obtained by performing a $y P_{4} x$-change and a $y \bar{P}_{4} x$-change, respectively. Then, $M_{12}\left(P_{N}^{\prime}\right)-$ $M_{12}\left(P_{N}\right)=\frac{1}{4}-0=\frac{1}{4}$ and $M_{12}\left(\bar{P}_{N}^{\prime}\right)-M_{12}\left(\bar{P}_{N}\right)=1-\frac{1}{4}=\frac{3}{4}$. SIND would imply that $M_{12}\left(P_{N}^{\prime}\right)-M_{12}\left(P_{N}\right)=M_{12}\left(\bar{P}_{N}^{\prime}\right)-M_{12}\left(\bar{P}_{N}\right)$.

Monotonicity: The cohesiveness measure $M_{4}$ satisfies REP, NEU, SIND, and FR. However, it is not monotonic.

Full Range: The cohesiveness measure $M_{5}$ satisfies REP, NEU, SIND, and MON. However, it does not satisfy FR. 